\title{
Tyrosine kinase FYN negatively regulates NOX4 in cardiac remodeling
}

\author{
Shouji Matsushima, ${ }^{1,2,3}$ Junya Kuroda, ${ }^{1}$ Peiyong Zhai, ${ }^{1}$ Tong Liu, ${ }^{4}$ Shohei Ikeda, ${ }^{1}$ Narayani Nagarajan, ${ }^{1}$ Shin-ichi Oka, ${ }^{1}$ \\ Takashi Yokota, ${ }^{2}$ Shintaro Kinugawa, ${ }^{2}$ Chiao-Po Hsu, ${ }^{5}$ Hong Li, ${ }^{4}$ Hiroyuki Tsutsui, ${ }^{2,3}$ and Junichi Sadoshima \\ 'Department of Cell Biology and Molecular Medicine, Cardiovascular Research Institute, Rutgers New Jersey Medical School, Newark, New Jersey, USA. ${ }^{2}$ Department of Cardiovascular Medicine, \\ Hokkaido University Graduate School of Medicine, Sapporo, Japan. ${ }^{3}$ Department of Cardiovascular Medicine, Kyushu University Graduate School of Medicinal Sciences, Fukuoka, Japan. ${ }^{4}$ Center for Advanced \\ Proteomics Research, Department of Biochemistry and Molecular Biology, Rutgers New Jersey Medical School, Newark, New Jersey, USA. ${ }^{5}$ Division of Cardiovascular Surgery, Department of Surgery, \\ Taipei Veterans General Hospital, National Yang-Ming University School of Medicine, Taipei, Taiwan.
}

NADPH oxidases (Noxes) produce ROS that regulate cell growth and death. NOX4 expression in cardiomyocytes (CMs) plays an important role in cardiac remodeling and injury, but the posttranslational mechanisms that modulate this enzyme are poorly understood. Here, we determined that FYN, a Src family tyrosine kinase, interacts with the C-terminal domain of NOX4. FYN and NOX4 colocalized in perinuclear mitochondria, ER, and nuclear fractions in CMs, and FYN expression negatively regulated NOX4-induced $\mathrm{O}_{2}$ - production and apoptosis in CMs. Mechanistically, we found that direct phosphorylation of tyrosine 566 on NOX4 was critical for this FYN-mediated negative regulation. Transverse aortic constriction activated FYN in the left ventricle (LV), and FYN-deficient mice displayed exacerbated cardiac hypertrophy and dysfunction and increased ROS production and apoptosis. Deletion of Nox4 rescued the exaggerated LV remodeling in FYN-deficient mice. Furthermore, FYN expression was markedly decreased in failing human hearts, corroborating its role as a regulator of cardiac cell death and ROS production. In conclusion, FYN is activated by oxidative stress and serves as a negative feedback regulator of NOX4 in CMs during cardiac remodeling.

\section{Introduction}

NADPH oxidases (Noxes) are the only known enzymes whose sole biological function is to purposefully produce $\mathrm{O}_{2}{ }^{-}$or $\mathrm{H}_{2} \mathrm{O}_{2}$, and they are major sources of ROS in the cardiovascular system (1). Among the seven members of the Nox family of proteins (NOX1-5 and DUOX1 and -2), NOX4 is abundantly expressed in cardiomyocytes (CMs) and plays a crucial role in the development of cardiac remodeling and injury. NOX4 localizes in the perinuclear region including the mitochondria, nucleus, and ER in CMs (2-4). Hypertrophic stimuli induce upregulation of NOX4 in mitochondria, thereby leading to increased production of ROS, mitochondrial dysfunction and apoptosis in CMs (2). NOX4 is also upregulated in the nucleus in response to phenylephrine and promotes hypertrophy through oxidation of class II histone deacetylases (3). Cardiac specific upregulation of human NOX4 stimulates cardiac fibrosis and hypertrophy in mice through activation of Akt/mTOR and NF- $\mathrm{KB}$ signaling (5). Cardiac hypertrophy and dysfunction in response to pressure overload (PO) were significantly attenuated in cardiac-specific, but not systemic, NOX4 knockout (KO) mice, and this was accompanied by a decrease in ROS production and preservation of mitochondrial function $(3,6,7)$.

Excess ROS production causes cell death in the heart and in CMs. Conversely, strictly regulated generation of ROS at low levels mediates physiological functions such as growth, differentiation, and metabolism in CMs $(8,9)$. Indeed, excessive suppression

Conflict of interest: The authors have declared that no conflict of interest exists. Submitted: November 17, 2015; Accepted: June 30, 2016.

Reference information: J Clin Invest. 2016;126(9):3403-3416. doi:10.1172/JCI85624. of Noxes below physiological levels can induce myocardial injury in response to ischemia/reperfusion (10). Suppression of NOX4 also exacerbates death of CMs during glucose starvation by inhibiting autophagy (4). These results suggest that the activity of Noxes must be maintained at appropriate levels for the survival of CMs. The mechanism of regulation of the classical phagocyte oxidase, NOX2, by cytosolic factors (p47phox $, \mathrm{p} 67^{\text {phox }}, \mathrm{p} 40^{\text {phox }}$, and Rac) (11, 12) has been well elucidated. On the other hand, NOX4 is believed to be constitutively active and its activity is controlled primarily by its expression levels $(13,14)$, and posttranslational modification of NOX4 has been poorly investigated.

We here demonstrate that FYN, a nonreceptor-type tyrosine kinase, directly interacts with NOX4 and phosphorylates tyrosine 566 in the C-terminus of NOX4. Through phosphorylation of NOX4, FYN negatively regulates NOX4 activity in CMs, thereby inhibiting apoptosis in the heart and preventing cardiac remodeling after PO. FYN is activated by NOX4, creating a negative feedback loop in the FYN-NOX4 interaction. We propose that FYN serves as a sensor of ROS and a negative feedback regulator of NOX4 in the heart.

\section{Results}

FYN interacts with NOX 4 in CMs. In order to identify a potentially novel mechanism regulating the activity of NOX4, we conducted yeast two-hybrid screenings using the C-terminal cytoplasmic domain of NOX4 (amino acids 406-578) (NOX4-CT) as bait and the human heart cDNA library. One of the clones we identified was FYN, a nonreceptor-type tyrosine kinase. We confirmed that recombinant NOX4-CT interacted with FYN in pull-down assays 
A

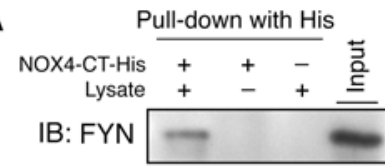

C

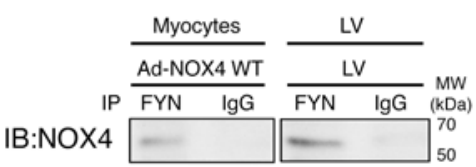

IB:NOX4

$5 \%$ input

D
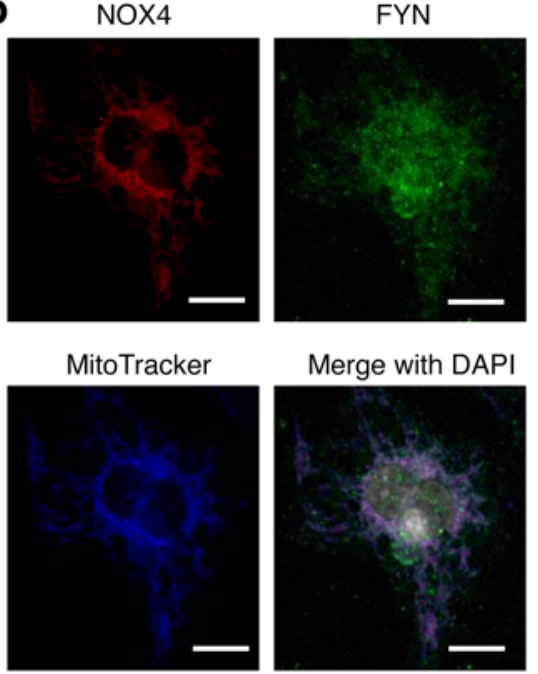

B

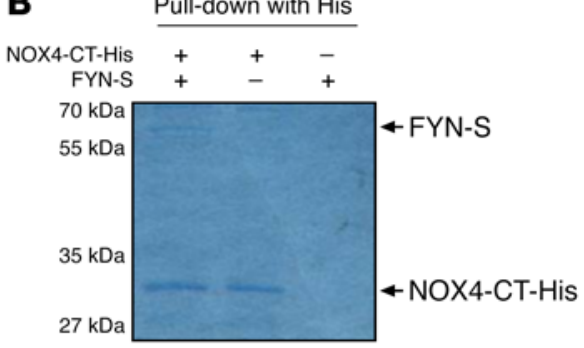

E

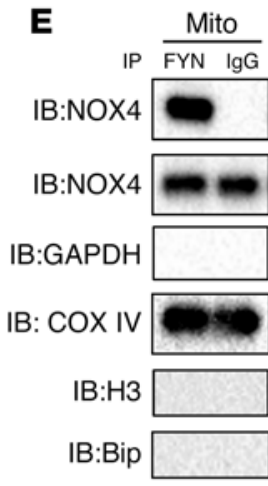

Figure 1. FYN interacts with NOX4 in CMs. (A) Interaction between the NOX4 C-terminus (NOX4-CT) and FYN was examined by pull-down assays with His-tagged NOX4-CT recombinant protein and whole cell lysates. IB, immunoblot. The experiment was conducted 3 times. (B) Interaction between the NOX4-CT and FYN was examined by pull-down assays using indicated recombinant proteins. (C) Coimmunoprecipitation assays using lysates of CMs transduced with Ad-NOX4 WT and heart homogenates. After immunoprecipitation with control IgC or a FYN antibody, immunoblotting for NOX4 was performed. Immunoblots of input controls (5\% lysates) are also shown. IP, immunoprecipitation. The experiment was conducted 3 times. (D) Cultured CMs were costained with anti-NOX4 antibody (red), anti-FYN antibody (green), MitoTracker (blue), and DAPI. Scale bar: $10 \mu \mathrm{m}$. The experiment was conducted 3 times. (E) Mitochondrial, ER, and nuclear fractions were prepared from cultured CMs. Coimmunoprecipitation assays in each fraction from CMs transduced with Ad-NOX4 WT. After immunoprecipitation with control IgC or a FYN antibody, immunoblotting for NOX4 was performed. Immunoblots of input controls (5\% lysates) are also shown. The purity of each fraction was confirmed by the absence or presence of GAP$\mathrm{DH}$, cytochrome C oxidase IV (COX IV), histone $\mathrm{H} 3$, and binding immunoglobulin protein (Bip). The experiment was conducted 3 times. after incubation of NOX4-CT with CM lysate (Figure 1A). Furthermore, NOX4-CT interacted with recombinant FYN in in vitro binding assays, suggesting that their interaction is direct (Figure 1B). Next, we investigated the molecular interaction between NOX4 and FYN in vivo. NOX4 was coimmunoprecipitated with endogenous FYN in NOX4-overexpressing CM lysates and WT mouse heart homogenates (Figure 1C). Coimmunoprecipitation of NOX4 was not observed when control IgG was used for immunoprecipitation. Immunostaining showed that FYN and NOX4 are costained in the perinuclear area and costained with MitoTracker, a mitochondrial indicator (Figure 1D). Furthermore, immunoprecipitation assays demonstrated colocalization of NOX4 and FYN in the mitochondrial, ER, and nuclear fractions (Figure 1E), suggesting that FYN and NOX4 interact with one another in mitochondria, the ER, and the nucleus.

FYN negatively regulates NOX4-induced ROS production and apoptosis. In order to elucidate how FYN affects the function of NOX4 in CMs, FYN was either overexpressed or downregulated by adenovirus-mediated transduction (Figure 2A). Overexpression or downregulation of FYN did not significantly affect the expression level of NOX4 in CMs (Figure 2A). However, $\mathrm{O}_{2}^{-}$production in the mitochondrial fraction, evaluated as the NADHor NADPH-dependent SOD-inhibitable component of lucigenin chemiluminescence, was attenuated by overexpression of FYN and enhanced by downregulation of FYN in both the presence and absence of NOX4 overexpression, suggesting that FYN negatively regulates NOX4 activity (Figure 2B and Supplemental
Figure 1A; supplemental material available online with this article; doi:10.1172/JCI85624DS1). Likewise, mitochondrial $\mathrm{H}_{2} \mathrm{O}_{2}$ production, as evaluated with Amplex Red assays, was decreased by overexpression of FYN and increased by downregulation of FYN (Supplemental Figure 1B). Importantly, downregulation of NOX4 abrogated the changes in mitochondrial $\mathrm{O}_{2}^{-}$production caused by overexpression or downregulation of FYN, whereas rotenone, a mitochondrial complex I inhibitor, did not affect FYN-induced modulation of mitochondrial $\mathrm{O}_{2}{ }^{-}$production, suggesting that NOX4, rather than the mitochondrial electron transport chain, mediates the effect of $\mathrm{FYN}$ upon $\mathrm{O}_{2}^{-}$production in CMs (Supplemental Figure 2).

1-Naphthyl PP1, a FYN inhibitor, increased $\mathrm{H}_{2} \mathrm{O}_{2}$ production in CMs (Supplemental Figure 3). Since FYN and NOX4 colocalize not only in mitochondria but also in the ER and nucleus, we also evaluated the ROS production in the ER and nuclear fractions. NOX4-derived $\mathrm{O}_{2}{ }^{-}$and $\mathrm{H}_{2} \mathrm{O}_{2}$ in the ER and nuclear fractions were also negatively regulated by FYN (Supplemental Figure 4, A-D). However, immunoblot analysis demonstrated that overexpression of FYN does not change the relative level of NOX4 in the mitochondrial, ER, and nuclear fractions, suggesting that FYN does not affect localization of NOX4 (Supplemental Figure 5A).

Since overexpression of NOX4 induces apoptosis in CMs (2), we examined whether FYN affects NOX4-induced cell death. TUNEL assays and evaluation of cleaved caspase-3 levels indicated that overexpression of FYN inhibited NOX4-induced apoptosis in CMs, whereas downregulation of FYN exacerbated it (Figure 
A
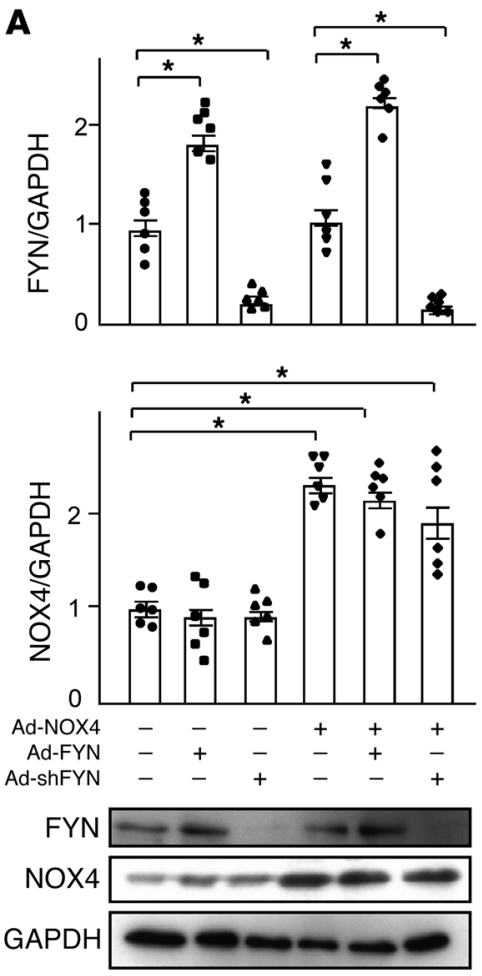

B

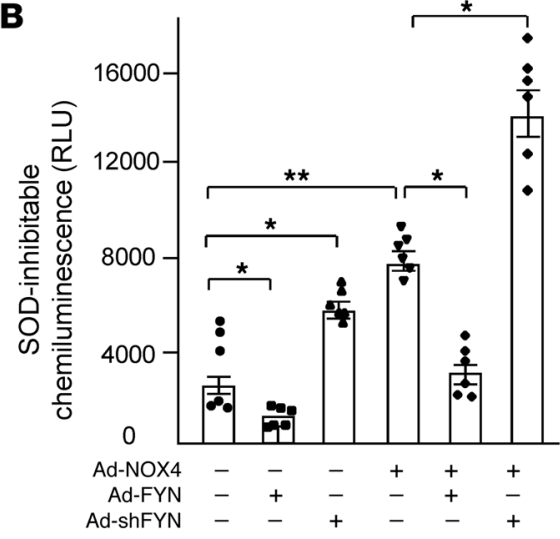

C

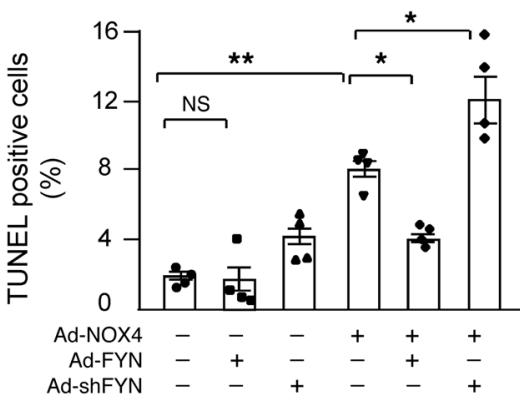

Figure 2. FYN negatively regulates NOX4-induced ROS production and apoptosis. (A) Protein levels of FYN, NOX4, and GAPDH in cultured neonatal rat CMs transduced with indicated adenoviruses $(n=6)$. The experiment was conducted 3 times. (B) NADPHdependent $\mathrm{O}_{2}{ }^{-}$release was measured by the lucigenin method. The SOD-inhibitable component of $\mathrm{O}_{2}$ release from the mitochondrial fraction in CMs transduced with indicated adenoviruses was determined $(n=6)$. The experiment was conducted 3 times. (C) Apoptosis in CMs transduced with indicated adenoviruses was evaluated with TUNEL staining $(n=4)$. The experiment was conducted 3 times. Statistical analyses were done by 1-way ANOVA followed by a post-hoc Fisher's comparison test. ${ }^{*} P<0.05$, ${ }^{*} P<0.01$. NS, not statistically significant.
2C and Supplemental Figure 6A), suggesting that FYN negatively affects NOX4-induced apoptosis in CMs.

NOX4 interacts with the N-terminal unique domain of FYN. The Src family of tyrosine kinases, including FYN, Src, and YES, have similar structures. We investigated whether Src and YES interact with NOX4. Immunoprecipitation assays showed that neither Src nor YES interacts with NOX4 in NOX4-overexpressing CMs (Figure 3A and Supplemental Figure 7). The observation that Src and YES failed to interact with NOX4 raised the possibility that a unique domain of FYN, which is not conserved among the Src family kinases, is critical for its binding to NOX4-CT. We generated S-protein-tagged recombinant proteins of FYN (amino acids 1-80), containing the unique domain, of FYN (amino acids 81-537), containing SH3, SH2, and kinase domains, and of FYN (amino acids 1-537), a full-length form of FYN. In vitro binding assays showed that $\mathrm{FYN}_{1-80}$ and FYN (1-537), but not FYN (81537), interact with NOX4-CT (Figure 3B). In order to determine whether $\mathrm{FYN}_{1-80}$ interacts with NOX4 in CMs, CMs were transduced with adenovirus harboring human influenza hemagglutinin (HA)-tagged $\mathrm{FYN}_{1-80}$. Coimmunoprecipitation assays revealed that $\mathrm{FYN}_{1-80}$ interacts with NOX4 (Figure 3C). Importantly, the NOX4 level in the supernatant after immunoprecipitation with an HA antibody (Figure 3C, indicated as "Lysate after IP") was markedly decreased in lysates prepared from myocytes transduced with adenovirus harboring $\mathrm{FYN}_{1-80}-\mathrm{HA}$. These results indicate that the unique domain of FYN mediates binding to NOX4 in CMs (Figure $3 \mathrm{C})$. They also suggest that, when found in excess, $\mathrm{FYN}_{1-80}$ interferes with the interaction between endogenous FYN and NOX4. In fact, $\mathrm{FYN}_{1-80}$ enhances ROS production in CMs, particularly in mitochondria, at baseline and in the presence of NOX4 overexpression, as evaluated with the SOD-inhibitable component of lucigenin chemiluminescence (Figure 3D) and with mitochondria-targeted HyPer, which allows sensitive measurement of $\mathrm{H}_{2} \mathrm{O}_{2}$ levels (Figure 3E), indicating that binding of FYN to NOX4 at this site is essential for negative regulation of NOX4 by FYN.

FYN phosphorylates tyrosine 566 in the C-terminus of NOX4. We then investigated whether FYN phosphorylates NOX4 in its C-terminus. In vitro kinase assays revealed that FYN phosphorylates NOX4-CT proteins (Figure 4A). Next, we identified the phosphorylation site in NOX4 using mass spectrometry (MS) analysis. After a FYN kinase reaction, the MS/MS spectrum showed a doubly charged ion corresponding to a phospho-peptide containing tyrosine 566 (Y566), suggesting that FYN phosphorylates NOX4 at Y566 (Figure 4B).

To test whether other tyrosine residues are also phosphorylated by FYN, we performed in vitro kinase assays. We prepared 6 peptides containing tyrosine residues in NOX4-CT (NOX4-CT-388, NOX4-CT-404-408, NOX4-CT-423, NOX4-CT-452, NOX4-CT480, NOX4-CT-566). Of these, only the Y566-containing peptide (NOX4-CT-566) was phosphorylated by FYN in vitro (Figure 4C).

To confirm that Y566 in NOX4 is phosphorylated by FYN in situ, we generated a polyclonal antibody specifically detecting NOX4 phosphorylated at Y566. NOX4 phosphorylated at Y566 was detected in lysates of NOX4-overexpressing CMs and in mitochondrial fractions from WT mouse hearts (Figure 4, D and E). Whereas overexpression of FYN increased phosphorylation of NOX4 at Y566, downregulation of FYN decreased it (Figure $4, \mathrm{D}$ and E). Y566 is conserved among rats, mice, and humans in NOX4, but it does not exist in NOX2 (Figure 4F).

Phosphorylation of tyrosine 566 negatively regulates NOX4. To elucidate the significance of phosphorylation of NOX4, we generated adenovirus harboring a Y566A mutant of NOX4 that can- 
A

$$
\text { IP } \frac{\text { Ad-NOX4 WT }}{\text { Frc YES IgG }}
$$

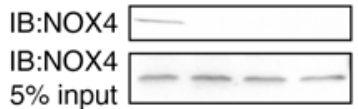

C

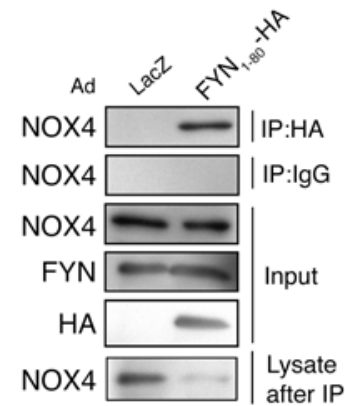

B

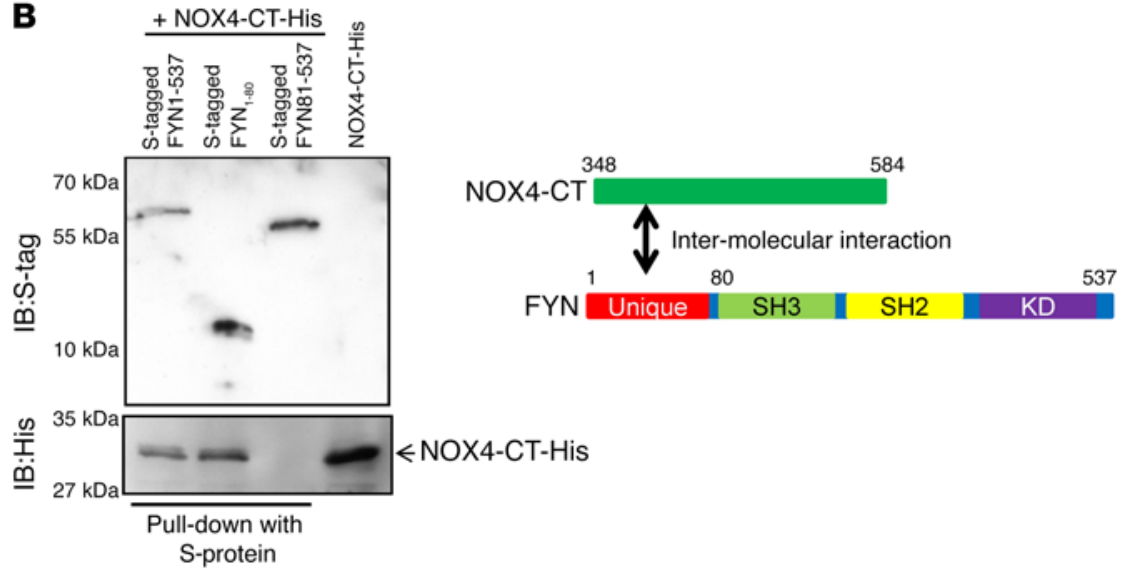

D

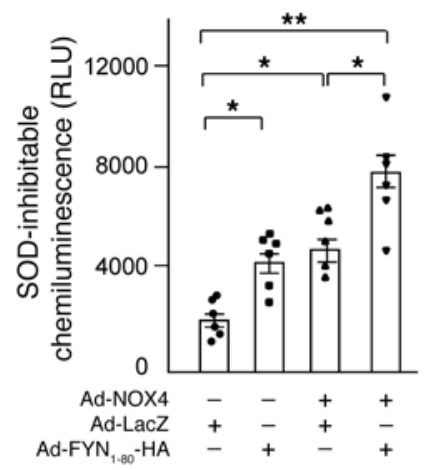

E

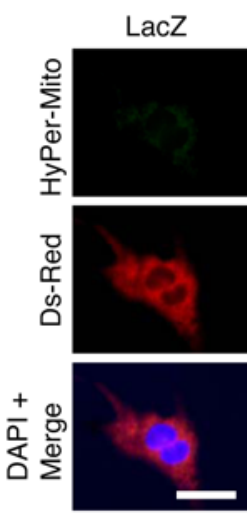

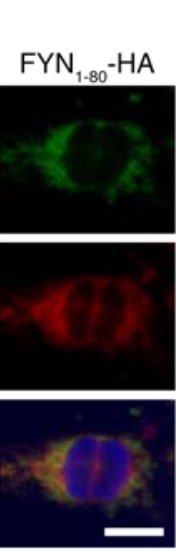

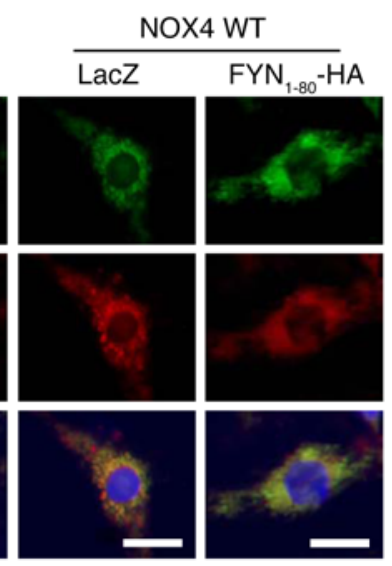

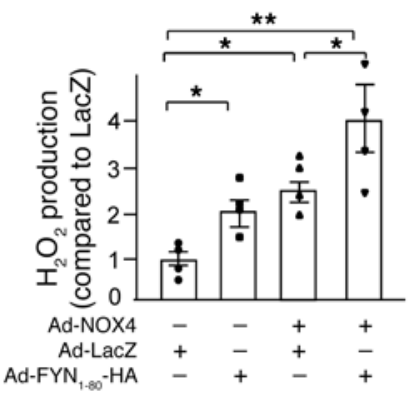

Figure 3. FYN 1-80, , but not Src or YES, interacts with NOX4 through binding between FYN 1-80 $_{\text {and }}$ and NOX4 C-terminus. (A) Coimmunoprecipitation assays using lysates of CMs transduced with Ad-NOX4 WT. After immunoprecipitation (IP) with control IgC or anti-FYN, anti-Src, or anti-YES antibody, immunoblotting for NOX4 was performed. Immunoblots of input controls (5\% lysates) are also shown. (B) The indicated S-protein-tagged proteins were incubated with His-tagged NOX4 C-terminus for pull-down assays (upper). Interaction was detected by immunoblots with an anti-His antibody (lower). (C) Coimmunoprecipitation assays using lysates of CMs transduced with the indicated adenoviruses. After immunoprecipitation with control IgC or an HA antibody, immunoblots for endogenous NOX4 were performed. Immunoblots of input controls (5\% lysates) and an immunoblot of remaining lysates after coimmunoprecipitation are also shown. (D) The SOD-inhibitable component of $\mathrm{O}_{2}^{-}$release from the mitochondrial fraction of $\mathrm{CMs}$ transduced with indicated adenoviruses was determined by the lucigenin method $(n=6)$. (E) CMs were transduced with Ad-HyPer-mito and Ad-Ds-Red, together with Ad-LacZ, Ad-FYN ${ }_{1-80}-\mathrm{HA}_{\text {, }}$ or Ad-NOX4 WT. Green (HyPer-mito) and red (Ds-Red) staining indicate $\mathrm{H}_{2} \mathrm{O}_{2}$ production and mitochondria, respectively. Nuclei were stained with DAPI (blue) $(n=4)$. Scale bar: $10 \mu \mathrm{m}$. Statistical analyses were done by 1-way ANOVA followed by a post-hoc Fisher's comparison test. ${ }^{*} P<0.05,{ }^{* *} P<0.01$.

not be phosphorylated by FYN and a Y566D mutant that mimics phosphorylation. Adenovirus-mediated transduction of CMs induced similar levels of expression of NOX4 and NOX4 Y566A (Figure $5 \mathrm{~A}$ ). $\mathrm{O}_{2}^{-}$production from the mitochondrial fraction of NOX4-overexpressing CMs, as evaluated with lucigenin chemiluminescence assays, was increased in an NADPH-dependent manner, but was significantly attenuated in the presence of FYN overexpression (Figure 5, B and $\mathrm{C}$ ). In contrast, $\mathrm{O}_{2}^{-}$production in the mitochondrial fraction of NOX4 Y566A-overexpressing CMs was not suppressed by overexpression of FYN (Figure 5, $\mathrm{B}$ and D). These results indicate that phosphorylation of NOX4 at Y566 inhibits NOX4 activity. Overexpression of NOX4 and NOX4 Y566A both increased TUNEL-positive CMs. Although FYN significantly reduced NOX4-induced increases in the number of TUNEL-positive CMs and the level of cleaved caspase-3, it did not do so in CMs transduced with adenovirus harboring NOX4 Y566A (Figure 5E and Supplemental Figure 6B). On the other hand, overexpression of the NOX4 Y566D mutant did not affect ROS production or cell survival compared to control (Supplemental Figure 8, A and B). NOX4 Y566A and NOX4 Y566D exhibited patterns of subcellular distribution in mitochondria, the nucleus, and the ER that were similar to those of the WT NOX4 (Supplemental Figure 5B), suggesting that phosphorylation of NOX4 at Y566 does not significantly change the subcellular localization of NOX4.

To further confirm the significance of phosphorylation of Y566 in NOX4 in vivo, adenovirus harboring LacZ, NOX4, or NOX4 Y566A was injected into the hearts of WT and cardiacspecific FYN transgenic [Tg(Myh6-Fyn), referred to here as Tg-FYN] mice. Expression of NOX4 increased $\mathrm{O}_{2}^{-}$production, the number of TUNEL-positive CMs, and the level of cleaved caspase-3 in WT mice, but these effects were attenuated in Tg-FYN mice. Although expression of NOX4 Y566A also increased $\mathrm{O}_{2}^{-}$ production, TUNEL-positive CMs, and cleaved caspase-3 in WT 
A

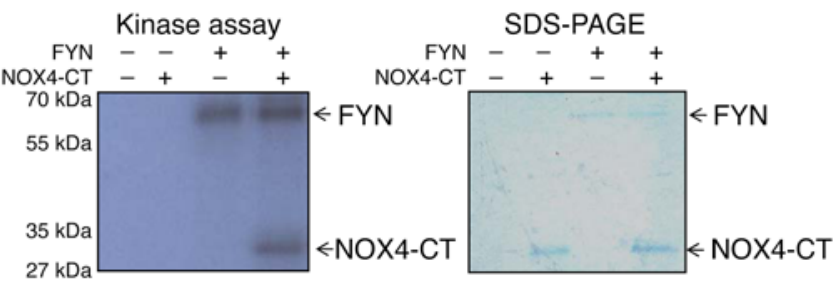

\section{B}

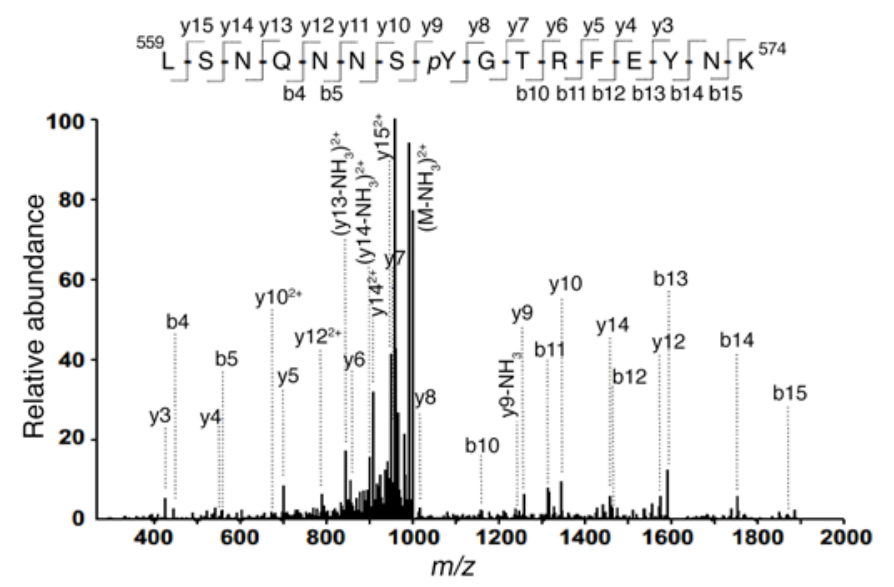

C

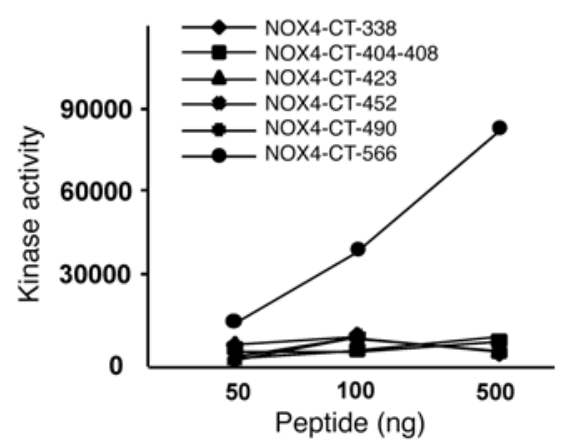

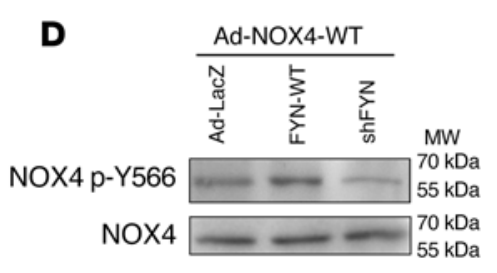

E

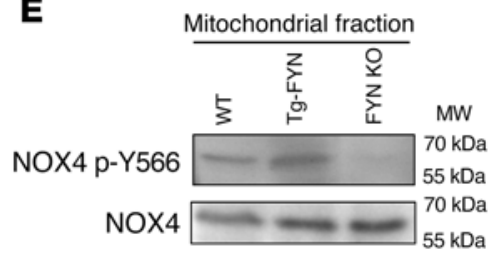

$\mathbf{F}$

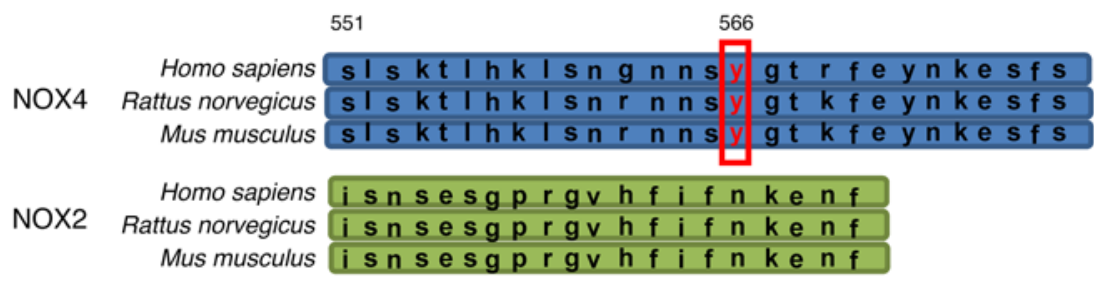

Figure 4. FYN phosphorylates Y566 in the NOX4 C-terminus. (A) Phosphorylation of NOX4 C-terminus by FYN was examined with in vitro kinase assays (left). Protein loaded in each group was resolved by SDS-PAGE (right). The experiment was conducted 2 times. (B) MS/MS spectrum of a doubly charged ion corresponding to phosphopeptide, ${ }^{559}$ LSNQNNSPYGTRFEYNK ${ }^{574}(\mathrm{~m} / \mathrm{z} 1,007.94)$ from NOX4. The observed $y$ - and b-ion series confirmed the peptide sequence and localization of phospho-Y566 with a Mascot score of 37. (C) Phosphorylation of the indicated fragments from the NOX4 C-terminus by FYN was examined by in vitro phosphorylation of 6 peptides containing tyrosine residues in NOX4-CT by FYN was examined with an in vitro kinase assay. The experiment was conducted 3 times. (D) Levels of phosphorylated NOX4 and NOX4 in cultured neonatal rat CMs transduced with indicated adenoviruses were examined by immunoblotting. The experiment was conducted 3 times. (E) Levels of phosphorylated NOX4 and NOX4 in the indicated mouse hearts were examined by immunoblotting. The experiment was conducted 3 times. (F) The sequence of the C-terminus in NOX4 (upper) and NOX2 (lower). The position of Y566 is indicated with a red rectangle.

mice, these were not attenuated in Tg-FYN mice (Figure 5, F and G, and Supplemental Figure 6C).

p22 2 hox physically interacts with NOX4 and is required for the enzymatic activity of NOX4. The N-terminal region (amino acids 6-11) of p22 $2^{\text {phox }}$, in particular, plays an important role in mediating NOX4 activation (15). p22 $2^{\text {phox }}$ has 2 glutamic acids (negatively charged amino acids) in its N-terminus (Supplemental Figure 9). Coimmunoprecipitation assays showed that both WT NOX4 and the NOX4 Y566A mutant interacted with $\mathrm{p} 22^{\text {phox }}$ abundantly. However, NOX4 Y566D exhibited a reduced affinity for $\mathrm{p} 22^{\text {phox }}$
(Figure 5H). In addition, the abundance of $\mathrm{p} 22^{\text {phox }}$ in CMs transduced with Ad-NOX4 Y566D was decreased compared to that in CMs transduced with either Ad-NOX4 WT or Ad-NOX4 Y566A (Figure $5 \mathrm{H}$ ). These results raise the possibility that the negatively charged C-terminus of NOX4 after Y566 phosphorylation may repel the $\mathrm{N}$-terminus of $\mathrm{p} 22^{\text {phox }}$ harboring negatively charged amino acids, thereby leading to inhibition of the interaction between NOX4 and p22 $2^{\text {phox }}$ and decreases in NOX4 activity (Figure 5I).

$F Y N$ is activated by oxidative stress. Src family tyrosine kinases are regulated in a redox-dependent manner (16). Thus, we inves- 

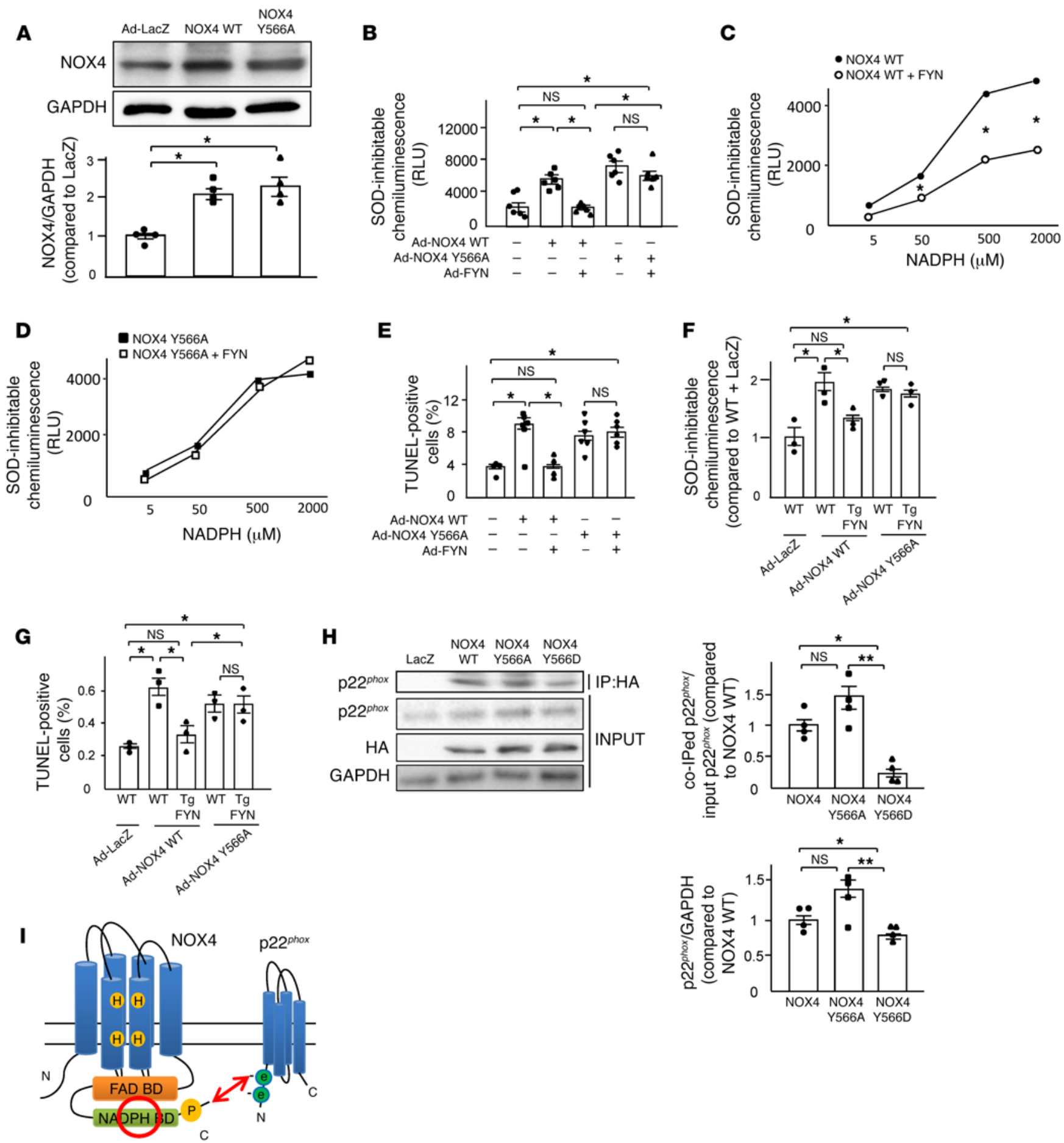

Figure 5. Phosphorylation of Y566 inhibits NOX4. (A) Protein levels of NOX4 and GAPDH in cultured neonatal rat CMs transduced with indicated adenoviruses $(n=4)$. (B) The NADPH-dependent SOD-inhibitable component of $\mathrm{O}_{2}$ - release from the mitochondrial fraction of CMs transduced with indicated adenoviruses was determined by the lucigenin method $(n=6)$. (C and $\mathbf{D})$ The SOD-inhibitable component of $\mathrm{O}_{2}^{-}$release from the mitochondrial fraction of CMs transduced with indicated adenoviruses in the presence of the indicated dose of NADPH was determined by the lucigenin method ( $n=6)$. (E) Apoptosis in CMs transduced with indicated adenoviruses was evaluated with TUNEL staining $(n=4-6)$. (F) The NADPH-dependent SOD-inhibitable component of $\mathrm{O}_{2}^{-}$release from the mitochondrial fraction from indicated mouse hearts was determined by the lucigenin method ( $n=3$ ). (G) Apoptosis in the indicated mouse hearts was evaluated with TUNEL staining $(n=3)$. (H) Upper: Coimmunoprecipitation assays of lysates of CMs transduced with the indicated adenovirus. After immunoprecipitation with an anti-HA antibody, immunoblots for endogenous p22 ${ }^{\text {phox }}$ were performed. Immunoblots of input controls (5\% lysates) are also shown. Lower: Protein levels of p22 ${ }^{\text {phox }}$ in CMs transduced with the indicated adenovirus. (I) A schematic figure showing that phosphorylation of Y566 in NOX4 inhibits interaction between NOX4 and p22 ${ }^{\text {phox }}$. The negatively charged C-terminus of NOX4 after phosphorylation may repel the $\mathrm{N}$-terminus of $\mathrm{p} 22^{\text {phox }}$ having negatively charged amino acids. Statistical analyses between groups were done by unpaired Student's $t$ test (C and D) or 1-way ANOVA followed by a post-hoc Fisher's comparison test (A, B, and $\mathbf{E}-\mathbf{H}) .{ }^{*} P<0.05,{ }^{* *} P<0.01$. 

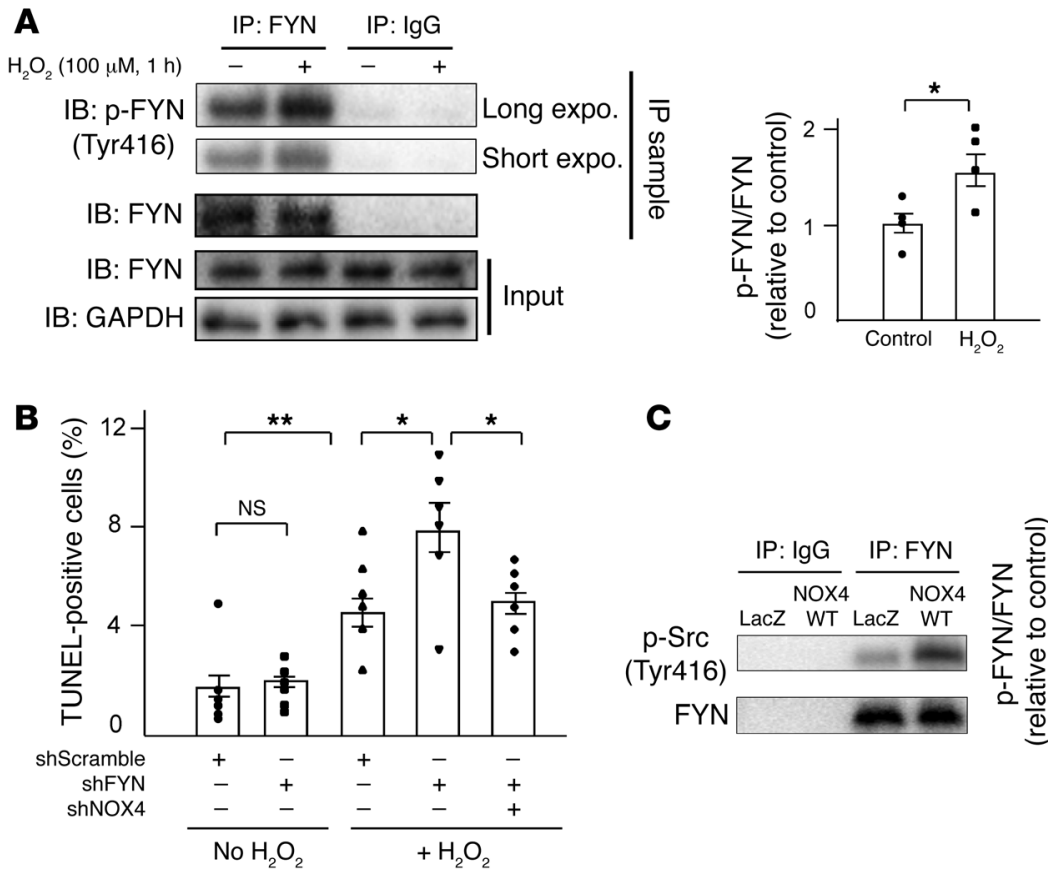

C
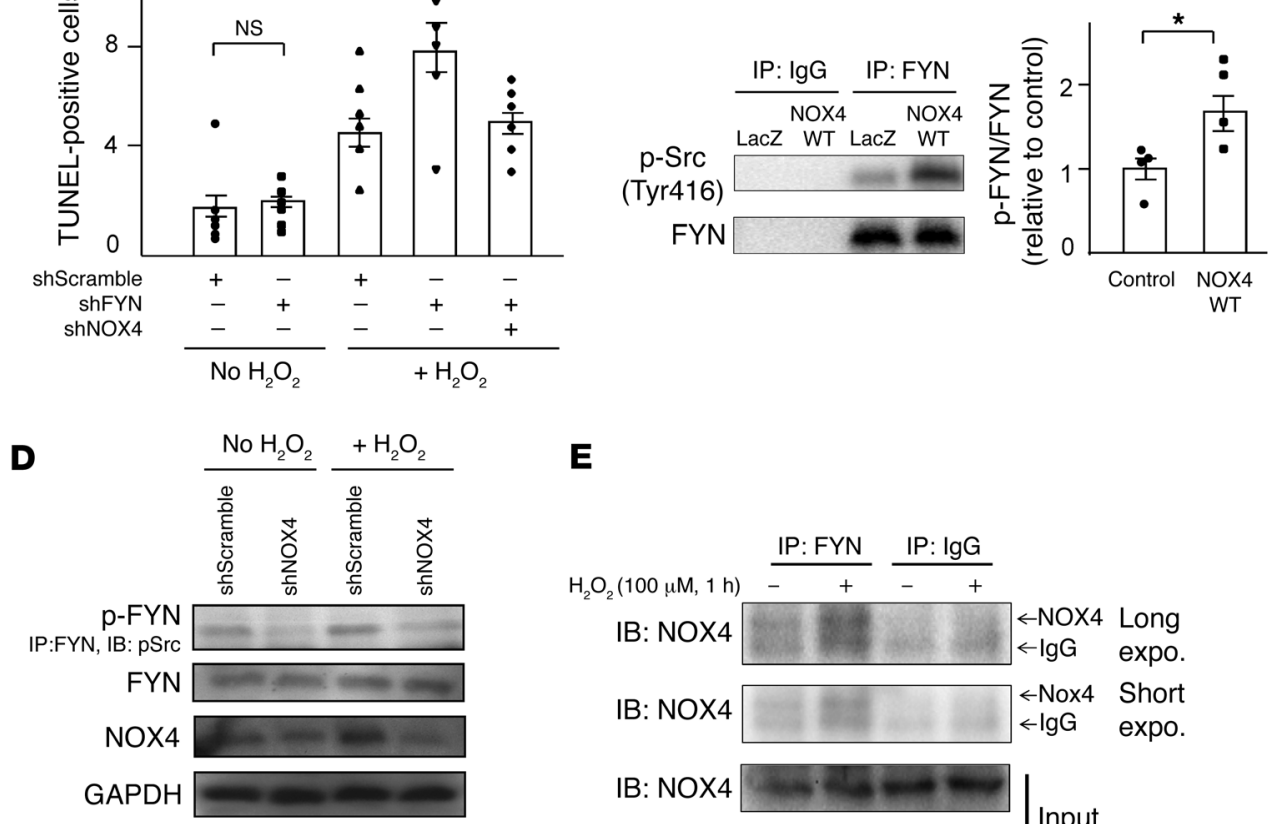

E

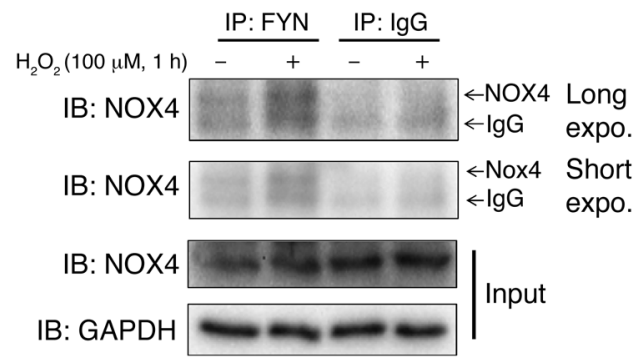

Figure 6. NOX4 and FYN affect the activity of one another. Cultured neonatal rat CMs were transduced with indicated adenoviruses. (A, B, and D) CMs were treated with or without $100 \mu \mathrm{M} \mathrm{H}_{2} \mathrm{O}_{2}$ for 1 hour. (A, C, and D) expression levels of phosphorylated FYN, total FYN, NOX4, and GAPDH were evaluated with immunoblotting. After immunoprecipitation (IP) with an anti-FYN antibody, immunoblot analyses (IB) for phospho-Src (S416) were performed to detect FYN phosphorylated at the tyrosine in the activation loop of the kinase domain ( $p-F Y N$ ). In the quantitative analysis, the level of p-FYN detected by the antiphospho-Src antibody/total FYN in control samples is expressed as 1 $(n=5)$. (B) Apoptosis in CMs transduced with indicated adenoviruses was evaluated with TUNEL staining $(n=6)$. (E) Coimmunoprecipitation assays with lysates of CMs treated with or without $\mathrm{H}_{2} \mathrm{O}_{2}$. After immunoprecipitation with control IgG and an anti-FYN antibody, immunoblot for endogenous NOX4 was performed. Immunoblots of NOX4 and GAPDH in input controls ( $5 \%$ lysates) are also shown. The experiment was conducted 3 times ( $\boldsymbol{D}$ and $\mathbf{E})$. Statistical analyses between groups were done by unpaired Student's $t$ test (A and $\mathbf{C}$ ) or 1-way ANOVA followed by a post-hoc Fisher's comparison test (B). ${ }^{*} P<0.05$, ${ }^{*} P<0.01$. tigated whether FYN is activated by oxidative stress in CMs and, if so, whether NOX4 is involved in activation of FYN. The activity of FYN was evaluated with tyrosine phosphorylation at Y416, which positively correlates with the activity of FYN. $\mathrm{H}_{2} \mathrm{O}_{2}$ induced phosphorylation of FYN at Y416, as determined by immunoblot analyses of anti-FYN immunoprecipitates with an antibody detecting Y416 phosphorylation of the Src family tyrosine kinases, indicating that oxidative stress activates FYN in CMs (Figure $6 \mathrm{~A})$. In order to test the functional significance of the FYN activation during $\mathrm{CM}$ apoptosis by oxidative stress, we investigated the effect of FYN downregulation upon $\mathrm{H}_{2} \mathrm{O}_{2}$-induced apoptosis. $\mathrm{CM}$ apoptosis in response to $\mathrm{H}_{2} \mathrm{O}_{2}$ was significantly enhanced by downregulation of FYN, whereas the enhancement was significantly suppressed by downregulation of NOX4 (Figure 6B), indicating that NOX4 is downstream of FYN and mediates $\mathrm{H}_{2} \mathrm{O}_{2}-$ induced apoptosis in CMs. Importantly, overexpression of NOX4 increased phosphorylation at Y416 in FYN (Figure 6C), whereas downregulation of NOX4 inhibited $\mathrm{H}_{2} \mathrm{O}_{2}$-induced activation of FYN in CMs (Figure 6D). In addition, Y416-phosphorylated FYN was decreased in $\mathrm{Nox}^{-1-}$ (NOX4 KO) mouse hearts compared to that in WT mouse hearts (Supplemental Figure 10). These results suggest that endogenous NOX4 acts also as an upstream regula- tor of FYN, thereby mediating activation of FYN. FYN physically interacts with NOX4 in CMs at baseline, and this interaction was enhanced in response to $\mathrm{H}_{2} \mathrm{O}_{2}$ treatment (Figure 6E). Taken together, these data show that NOX4 and FYN affect the activity of one another.

Deletion of FYN exacerbates cardiac hypertrophy and dysfunction in response to PO by stimulating NOX4. We have shown previously that expression of NOX4 in CMs is upregulated in response to PO, which in turn promotes hypertrophy and cardiac dysfunction in response to $\mathrm{PO}$ (3). To investigate the role of endogenous FYN in regulating NOX4 during $\mathrm{PO}$, we conducted transverse aortic constriction (TAC) in WT and Fyn ${ }^{-1}$ (FYN KO) mice. Protein expression of NOX 4 was increased 1,2, and 4 weeks after TAC in WT mice (Figure 7A). Interestingly, although FYN increased until 2 weeks after TAC, it decreased to the baseline level 4 weeks after TAC (Figure 7A). At 6 weeks after TAC, the NOX4 expression level in the mitochondrial, ER, and nuclear fractions was higher, whereas FYN was lower, than the basal level (Supplemental Figure 11A). Consistent with these changes, $\mathrm{O}_{2}^{-}$production in each fraction was increased 6 weeks after TAC (Supplemental Figure 11B).

Two weeks after TAC, total FYN, Y416 phosphorylation of FYN, and Y566 phosphorylation of NOX4 were increased in WT 
A

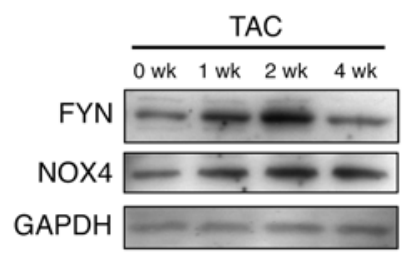

B

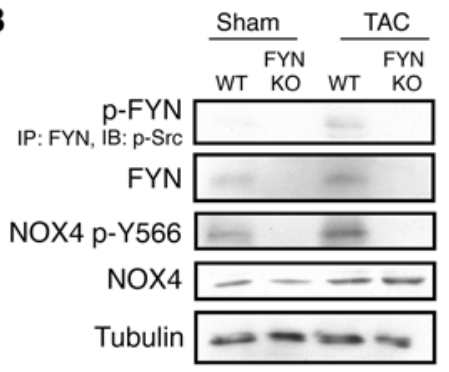

C

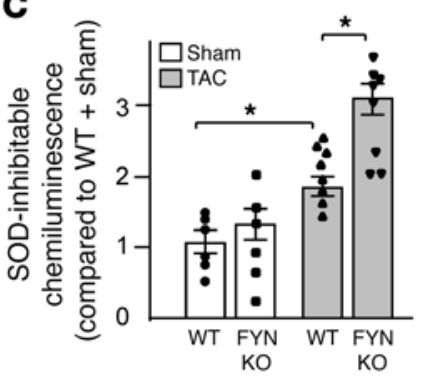

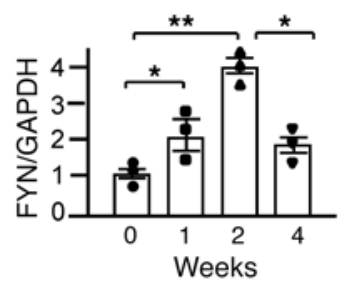
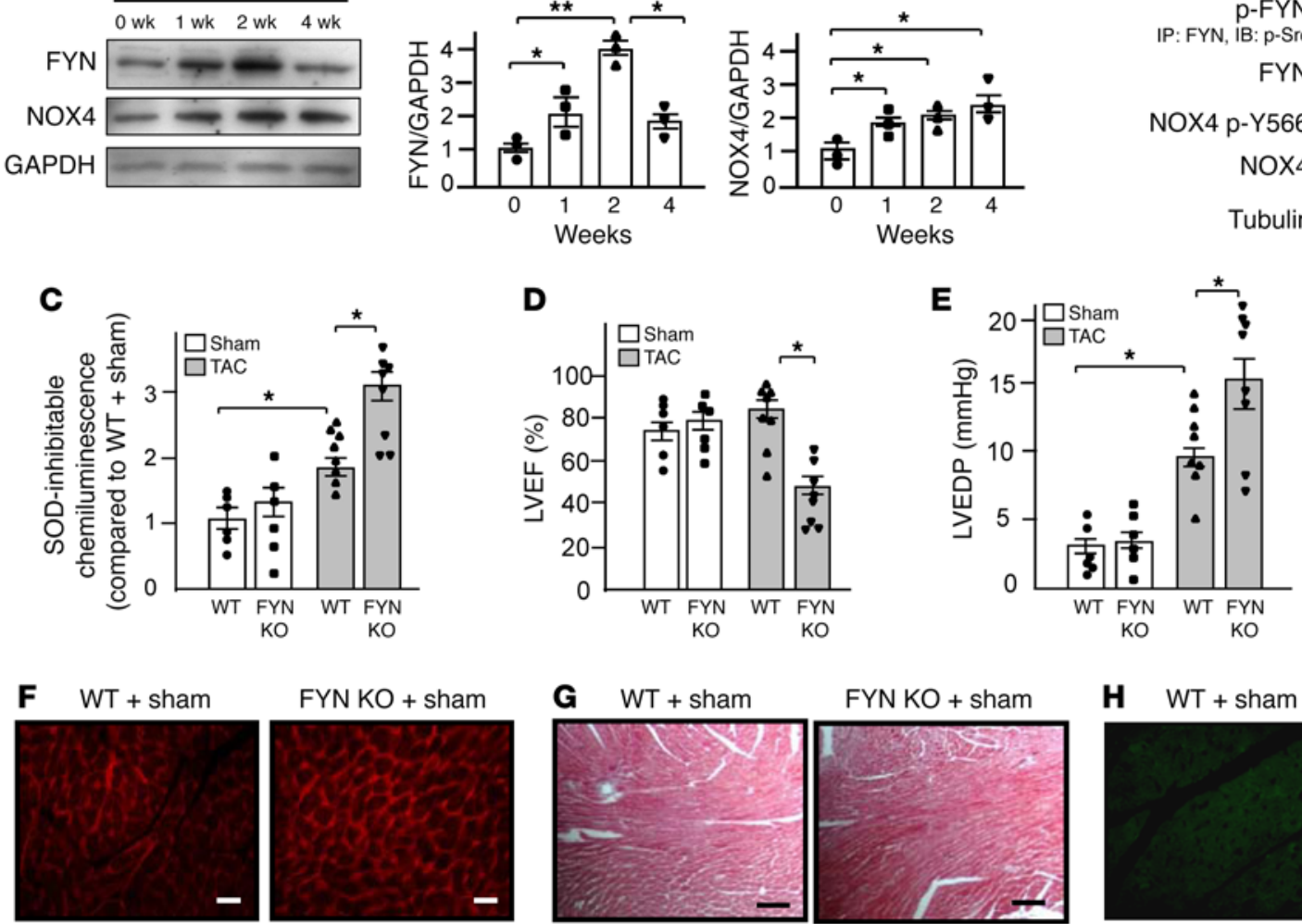

FYN KO + sham
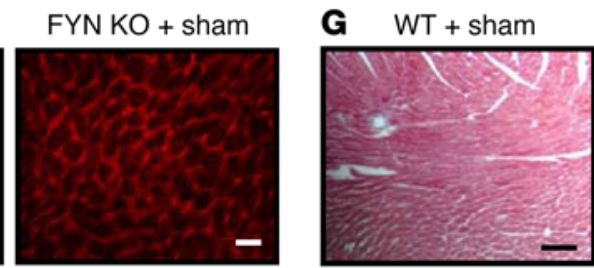

FYN KO + sham

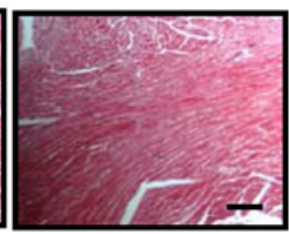

FYN KO + TAC
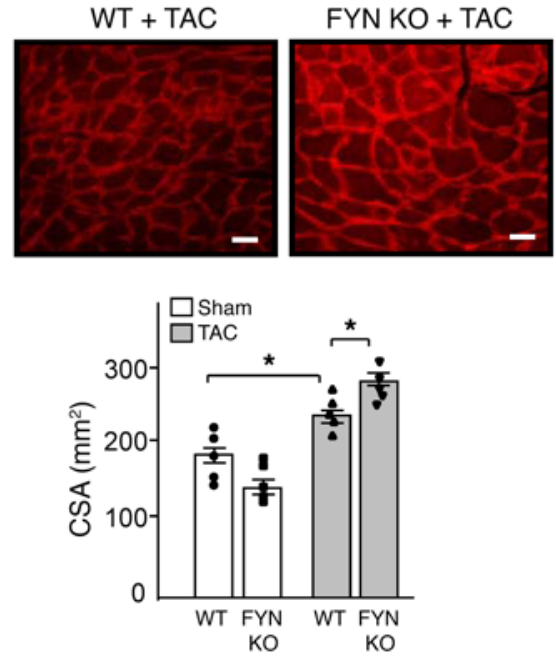
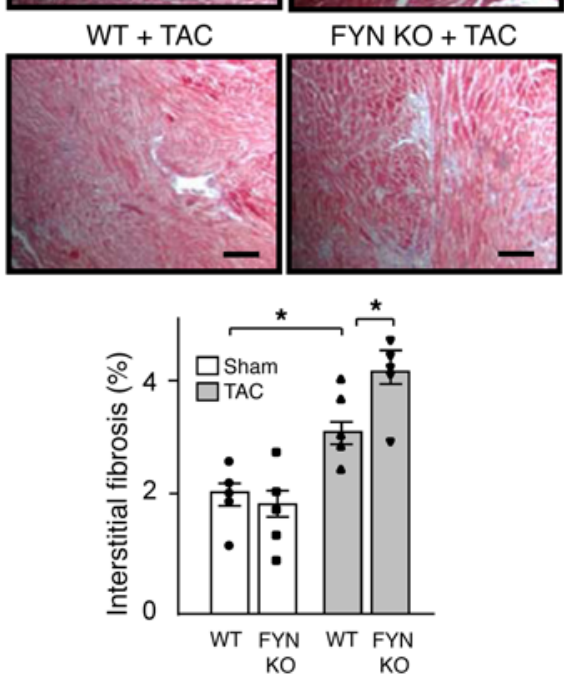
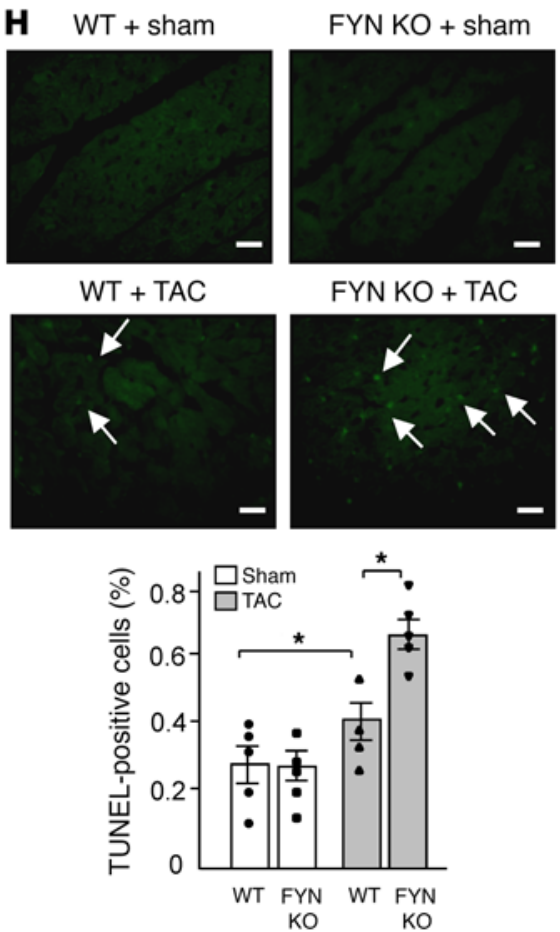

Figure 7. FYN KO mice exhibit exacerbation in cardiac remodeling after PO. (A) Protein levels of NOX4, FYN, and GAPDH in LV after TAC at various time points $(n=3)$. In B-H, all analyses were conducted 2 weeks after sham or TAC. (B) Protein levels of phospho-FYN, FYN, phospho-NOX4, NOX4, and GAPDH in LV of WT mice and FYN KO mice. After immunoprecipitation with an anti-FYN antibody, immunoblot analyses with phospho-Src (S416) antibody were performed to detect $\mathrm{p}-\mathrm{FYN}$. The experiment was conducted 3 times. (C) NADPH-dependent $\mathrm{O}_{2}^{-}$release was measured by the lucigenin method. The SOD-inhibitable component of $\mathrm{O}_{2}^{-}$release from the mitochondrial fraction in LV of WT mice and FYN KO mice is shown ( $\left.n=6-8\right)$. (D) LV ejection fraction (LVEF) was evaluated with echocardiography $(n=6-8)$. (E) LVEDP was evaluated with a Millar catheter $(n=6-8)$. (F) LV CM cross-sectional area (CSA) evaluated with wheat germ agglutinin staining $(n=5)$. Scale bar: $10 \mu \mathrm{m}$. (G) Interstitial fibrosis in the LV evaluated using Masson trichrome staining $(n=5)$. Scale bar: $50 \mu \mathrm{m}$. (H) Apoptosis in LV evaluated with TUNEL staining $(n=5)$. Scale bar: $10 \mu \mathrm{m}$. White arrows indicate apoptotic cells. Statistical analyses were done by 1-way ANOVA followed by a post-hoc Fisher's comparison test. ${ }^{*} P<0.05,{ }^{* *} P<0.01$.

mouse hearts but not in FYN KO mouse hearts (Figure 7B). Consistent with the differences in the level of Y566 phosphorylation, which negatively regulates the activity of NOX4, TAC-induced increases in $\mathrm{O}_{2}^{-}$production were exacerbated in FYN KO mice
(Figure 7C). TAC-induced increases in the ratio of $\mathrm{LV}$ weight/tibia length (LVW/TL) were also enhanced in FYN KO mice 2 weeks after TAC (Supplemental Figure 12A). Although the lung weight (LW)/TL ratio was maintained in WT mice 2 weeks after TAC, it 

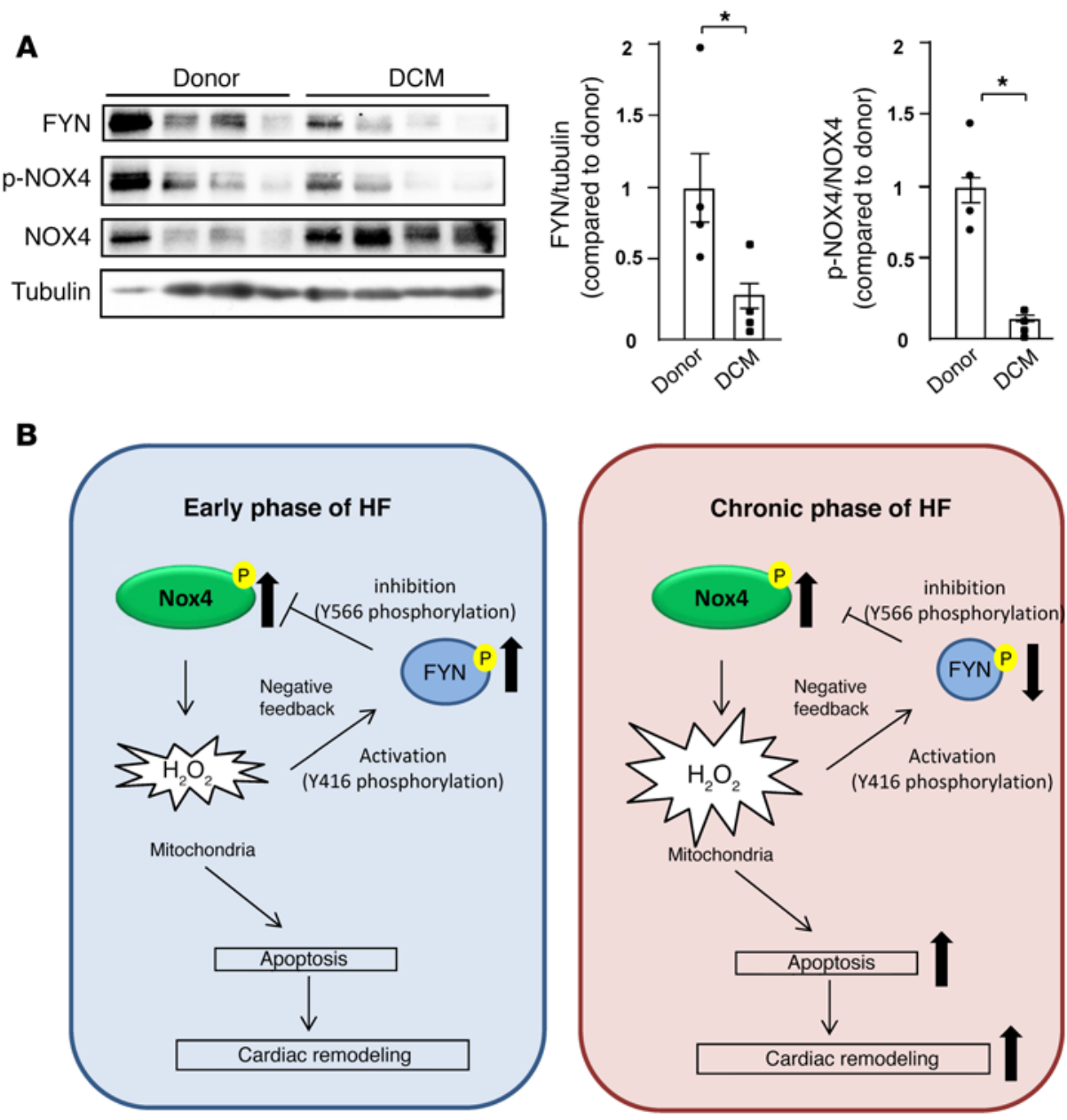

Figure 8. FYN is downregulated in failing human hearts. (A) Protein levels of FYN phosphorylated NOX4 at Y566 (p-NOX4), NOX4, and tubulin in hearts from normal donors and from patients with dilated cardiomyopathy $(n=4)$. (B) A schematic representation of the role of FYN in regulating NOX4 activity and mitochondrial ROS in early and chronic phases of heart failure (HF). Statistical analyses between groups were done by unpaired Student's $t$ test. ${ }^{*} P<0.05$. was significantly increased in FYN KO mice (Supplemental Figure 12B). Although LV ejection fraction was maintained in WT mice 2 weeks after TAC, it was decreased significantly in FYN KO mice, as evaluated with echocardiographic measurements (Figure 7D). The LV end-diastolic dimension and LV wall thickness were increased in FYN KO mice (Supplemental Table 1). Hemodynamic measurement indicated that LV end-diastolic pressure (LVEDP) was significantly elevated in WT mice 2 weeks after TAC and it was further elevated in FYN KO mice (Figure 7E). TAC-induced decreases in LV contractility and relaxation (the maximum and minimum derivatives of left ventricular pressure: $+/-\mathrm{dP} / \mathrm{dt}$ ) were exacerbated in FYN KO mice compared to in WT mice (Supplemental Table 2). Histological analyses showed that CM cross-sectional area and interstitial fibrosis were increased in the hearts of WT mice after TAC, and were further enhanced in FYN KO mice (Figure 7, F and G). Apoptosis, evaluated with both TUNEL assays and the level of cleaved caspase-3, was also increased in WT mice, and was further increased in FYN KO mice, after TAC (Figure 7H and Supplemental Figure 6D).

We hypothesized that the increase in TAC-induced cardiac hypertrophy and LV dysfunction in FYN KO mice is mediated through stimulation of NOX4. To test this hypothesis, we crossed FYN KO mice and cardiac-specific NOX4 KO (Nox $4^{f l / f l}$ Myh6-Cre, referred to here as cNOX4 $\mathrm{KO}$ ) mice to generate FYN KO-cNOX4 KO mice. FYN KO-cNOX4 KO mice did not show any appar- ent cardiac abnormality or changes in blood pressure at baseline (Supplemental Figure 13A). Two weeks after TAC, however, TACinduced increases in LVW/TL and LW/TL were attenuated in FYN KO-cNOX4 KO mice compared to those in FYN KO mice (Supplemental Figure 13, B and C). Echocardiographic analyses showed that simultaneous downregulation of NOX4 and FYN normalizes the TAC-induced increases in LV end-diastolic and end-systolic dimensions, increases in LV wall thickness, and decreases in LV ejection fraction observed in FYN KO mice (Supplemental Table 3). TAC-induced increases in LVEDP and decreases in $\pm \mathrm{dP} / \mathrm{dt}$ were also attenuated in FYN KO-cNOX4 KO mice compared to in FYN KO mice (Supplemental Figure 13D and Supplemental Table 4). In addition, TAC-induced increases in $\mathrm{O}_{2}^{-}$production in mitochondria, as evaluated with lucigenin chemiluminescent assays and in the number of TUNEL-positive CMs, were attenuated in FYN KO-cNOX4 KO mice compared to those in FYN KO mice (Supplemental Figure 13, E and F). These results suggest that NOX4 in CMs plays an important role in mediating the enhancement of cardiac hypertrophy and LV dysfunction observed in FYN KO mice.

Cardiac-specific overexpression of FYN attenuates ROS production, apoptosis, and cardiac dysfunction in response to $P O$. To elucidate the effect of FYN on NOX4-induced exacerbation of cardiac remodeling, we generated bigenic mice by crossing cardiac-specific Tg-FYN mice with cardiac-specific NOX4 transgenic [ $\operatorname{Tg}(M y h 6-$ Nox4), referred to here as Tg-NOX4] mice. We confirmed that 
FYN was elevated in Tg-FYN and in the bigenic mice and that NOX4 was elevated in Tg-NOX4 and in the bigenic mice (Supplemental Figure 14A). We then performed TAC operations on WT, Tg-NOX4, Tg-FYN, and bigenic mice. TAC-induced increases in ROS and cell death and decreases in cardiac function were exacerbated in Tg-NOX4, whereas they were attenuated in Tg-FYN, compared to those in WT mice (Supplemental Figure 14, B and C, and Supplemental Tables 5 and 6). Furthermore, overexpression of FYN in the bigenic mice attenuated the NOX4-induced exacerbation of CM hypertrophy (Supplemental Figure 14D) and cardiac dysfunction (Supplemental Figure 14, E and F, and Supplemental Tables 5 and 6) observed in Tg-NOX4 during PO. These data support our hypothesis that FYN negatively regulates NOX4-induced exacerbation of pathological hypertrophy.

FYN is downregulated in the failing human heart. We next examined whether the levels of FYN, NOX4, and NOX4 phosphorylated at Y566 are altered in the failing human heart, using heart samples obtained from patients with dilated cardiomyopathy (DCM) and from donors with normal hearts. The level of FYN was significantly lower in DCM hearts than in control hearts (Figure 8, A and B). The level of NOX4 was significantly higher in DCM hearts than in control hearts, whereas the level of NOX4 phosphorylated at Y566 was lower (Figure 8, A and B). Importantly, the ratio of phosphorylated NOX4 to total NOX4 was decreased in DCM hearts. These data suggest that negative regulation of NOX4 activity by FYN may be attenuated in DCM hearts.

FYN regulates NOX4 activity in physiological conditions. We have shown previously that NOX4-induced ROS production in mitochondria, the nucleus, and the ER affects the oxidation and function of aconitase 2 (2), HDAC4 (3), and Perk (4), respectively. NOX4 also upregulates Nrf2 in the nucleus (17). We therefore evaluated whether FYN affects the targets of NOX4 in each subcellular fraction. Although overexpression of NOX4 increased cysteine oxidation of HDAC4 and aconitase 2, as evaluated with iodoacetamide assays, these changes were attenuated by overexpression of FYN (Supplemental Figure 15). NOX4-induced phosphorylation of Perk at Thr980 was also inhibited by overexpression of FYN (Supplemental Figure 15). In addition, FYN prevented an increase in nuclear Nrf2 caused by NOX4 (Supplemental Figure 15). These results suggest that FYN negatively regulates the known downstream targets of NOX4. NOX4-derived ROS are known to serve as physiological mediators during glucose deprivation (GD). As shown previously (4), GD induced $\mathrm{O}_{2}^{-}$production in the ER fraction, which was decreased by overexpression of FYN and increased by downregulation of FYN. On the other hand, downregulation of NOX4 attenuated the regulation of $\mathrm{O}_{2}^{-}$production by FYN (Supplemental Figure 16A). Overexpression of FYN decreased the LC3-II level and cell survival in CMs during GD, whereas downregulation of FYN increased them (Supplemental Figure 16, B and C). GD increased phosphorylated FYN, representing an active form of the protein (Supplemental Figure 16D). These data indicate that FYN controls autophagy and cell survival by regulating NOX4 activity during GD.

\section{Discussion}

We here demonstrate that the tyrosine kinase FYN negatively regulates the ROS-producing activity of NOX4 through direct interaction with and phosphorylation of NOX4 in CMs. FYN is upregulated in response to $\mathrm{PO}$, and phosphorylation of Y566 in the C-terminus of NOX4 by FYN negatively regulates oxidative stress and cardiac cell death. Thus, FYN acts as a negative feedback regulator of NOX4-derived ROS, thereby inhibiting pathological hypertrophy in the heart.

In general, excessive ROS production in cells is harmful, as can be seen in several pathological conditions, by damaging DNA, protein, and lipids, and causing cell death $(8,9,18)$. Electron spin resonance shows that the level of $\mathrm{O}_{2}^{-}$is increased in the failing heart (19). Although there are several sources of ROS, NOX4 is a major source of ROS production in the heart and is involved in apoptosis in CMs in response to hypertrophic stimuli $(3,6)$. NOX4 localizes in intracellular membranes in mitochondria, ER, and the nucleus, and NOX4-derived ROS oxidize mitochondrial proteins, such as aconitase 2, citrate synthase, and NADH dehydrogenase, as well as nuclear proteins, including HDAC4, leading to CM apoptosis and hypertrophy, respectively $(3,6)$. Cardiac-specific, but not systemic, NOX4 KO mice show attenuation of mitochondrial dysfunction and cardiac remodeling after PO $(3,6,7)$. On the other hand, NOX2 is not essential for the development of cardiac hypertrophy after PO (20). NOX1, expressed mainly in the endothelial cells in the heart, is involved in endotoxin-induced apoptosis in CMs (21). These findings indicate that Noxes have isoform- and cell type-specific functions in the heart and that NOX4 in CMs is a promising target for treatment of cardiac remodeling and heart failure in response to PO.

Unlike other Nox isoforms, NOX4 is believed to be constitutively active. Whereas the activity of NOX1 and NOX2 is modulated by cytosolic factors, the activity of NOX4 is regulated primar-

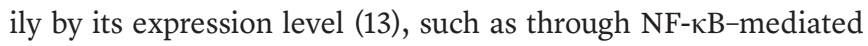
transcription $(3,4)$. Recently, Poldip2 was reported to be a novel positive regulator of NOX4 through its binding with NOX4 and p22 $2^{\text {phox }}$, thereby affecting cell migration in vascular smooth muscle cells (22). Elucidating mechanisms of posttranslational modification of NOX4 may allow identification of specific approaches to regulating the activity of NOX4. In the present study, we found that NOX4 and the tyrosine kinase FYN colocalize in the perinuclear area of CMs, including in the mitochondria, ER, and nucleus, and that the C-terminus of NOX4 interacts with FYN. NOX 4 is a 6 transmembrane domain-containing protein whose cytosolic portion (amino acids 305-578) has thirteen tyrosines. Among these tyrosines, only tyrosine 566 can be phosphorylated by FYN, leading to inactivation of NOX4 and inhibition of apoptosis. Tyrosine 566 in NOX4 is evolutionarily conserved in mammals, whereas NOX2 has no homologous tyrosine in its C-terminus (Figure 4F). To the best of our knowledge, FYN is the first molecule to be identified as a direct negative regulator of NOX4, acting through posttranslational modification.

FYN is a member of the Src family of nonreceptor tyrosine kinases, which is associated with molecular signaling in the immune system (23), neural system (24), energy metabolism (25), and cancer (26). FYN prevents apoptosis and promotes cell survival through phosphorylation of phosphatidylinositol 3-kinase enhancer-activating Akt (27). Src family kinases have been reported to be involved in mediating the cellular responses to ROS. Platelet exposure to $\mathrm{H}_{2} \mathrm{O}_{2}$ induces activation of Src, the prototype 
Src family kinase, by a protein kinase C-dependent pathway (28). YES, another Src family kinase, is known to be a downstream target of Nox in hepatocytes (29). However, it has not been clear whether Src family kinases regulate ROS production. We here demonstrated that FYN directly interacts with the NOX4 C-terminus and phosphorylates Y566 of NOX4. Phosphorylation of NOX4 at Y566 inhibits the function of NOX4, leading to a decrease in $\mathrm{O}_{2}{ }^{-}$production, whereas the Y566A mutation makes NOX4 insensitive to FYN, indicating that FYN regulates NOX4 through phosphorylation of Y566. Other Src family kinases, including Src and YES, appear not to interact with or affect the activity of NOX4. This is most likely because FYN interacts with the NOX4 C-terminus through its unique domain, which is not conserved in other Src family kinases.

The activity of the Src family kinases is tightly regulated by inhibitory phosphorylation of tyrosine 527 in the C-terminus. This phosphorylation induces an inactive conformation in the kinase through intramolecular binding with its $\mathrm{SH} 2$ domain. The inactive (closed) form is converted to the active (open) form through dephosphorylation of tyrosine 527 (30). This conformational change promotes autophosphorylation of tyrosine 416, leading to activation of the Src kinase (31). In addition, it has been reported recently that Src is oxidized at cysteines 245 and 487 in response to stimuli, and this redox-sensitive modification enhances kinase activity (32). Since these 2 cysteines are conserved in FYN, it will be interesting to test whether activation of FYN by oxidative stress is mediated through oxidation of these 2 cysteines, and, if so, whether it is mediated by NOX4-derived ROS. In any case, given (a) that physical interaction between NOX4 and FYN is increased in the presence of oxidative stress, (b) that $\mathrm{H}_{2} \mathrm{O}_{2}$-induced activation of FYN is mediated through NOX4-dependent mechanisms, and (c) that FYN negatively regulates the ROS-producing activity of NOX4, it appears that NOX4 and FYN form a complex in which NOX4 activates FYN, and FYN, in turn, acts as negative feedback regulator of NOX4.

How does phosphorylation of NOX4 decrease its activity? The membrane protein $\mathrm{p} 22^{\text {phox }}$ forms a heterodimeric complex with Noxes and is required for their catalytic activity. Recently, using mutational analysis, a dual tryptophan motif contained in the N-terminal region of $\mathrm{p} 22^{\text {phox }}$ was reported to be essential for NOX 4 activity (15). p22 $2^{\text {phox }}$ has 2 glutamic acids in its N-terminus. WT NOX4 and the NOX4 Y566A mutant can both interact with $\mathrm{p} 22^{\text {phox }}$, whereas the NOX4 Y566D mutant exhibits weaker interaction. These findings suggest that the phosphorylated, negatively charged C-terminus of NOX4 may repel the $\mathrm{N}$-terminus of $\mathrm{p} 22^{\text {phox }}$ containing negatively charged amino acids, thereby causing a decrease in interaction between NOX4 and p22 phox and in NOX4 activity.

Endogenous FYN negatively regulates ROS production in the heart after PO. Interestingly, the protein level of FYN in the heart increases 1 or 2 weeks after TAC (the early phase of cardiac remodeling), but reverts to the basal level by 4 weeks after TAC (the late phase of cardiac remodeling) in mice, and FYN is downregulated in end-stage failing hearts in humans. On the other hand, the level of NOX 4 increases at 1 week, and this increase persists until 4 weeks after TAC. Thus, both upregulation of NOX4 and insufficient upregulation of FYN may contribute to the increased ROS production in failing hearts (Figure $8 \mathrm{C}$ ). Downregulation of
FYN exacerbates NOX4-induced apoptosis in CMs. Consistent with this, FYN KO mice exhibited an increase in apoptosis in the heart after PO, which was attenuated by concomitant deletion of NOX4. Downregulation of FYN appears to facilitate the transition from preserved systolic function to decreased systolic function by stimulating production of ROS by NOX4 in the heart. Conversely, Tg-FYN mice showed decreases in ROS production and cell death and improvement of cardiac function compared with WT mice. Moreover, while Tg-NOX4 mice exhibited worse cardiac dysfunction and further increases in ROS production and apoptosis, overexpression of FYN attenuated the deleterious effects of NOX4 overexpression during PO. These results suggest that cell death and cardiac remodeling are critically regulated by a FYN-NOX4 axis in the heart during PO.

The mechanism by which FYN is regulated in failing hearts is not clear. Akt is known to be a downstream target of FYN, but has also been reported to be upstream of FYN (33). We confirmed that the time course of expression of Akt paralleled that of FYN after TAC (Supplemental Figure 17). Thus, Akt may be a regulator of FYN in failing hearts. In addition, it has recently been reported that microRNA-125a-3p negatively regulates FYN expression (34). Further experiments are required to address this question.

The role of NOX4 during PO is controversial. Our previous study demonstrated that NOX4 was detrimental (6), whereas Zhang et al. demonstrated that NOX4 is protective against PO (7). There a number of possible explanations for this discrepancy. One of the most important differences between the studies is the mode of deletion of NOX4 (cardiac-specific vs. systemic). We used cardiac-specific NOX4 KO mice and investigated the role of NOX4 in CMs. On the other hand, studies using systemic NOX4 KO mice cannot distinguish the effect of NOX4 in CMs from its effect in non-CMs. NOX4 enhances angiogenesis under ischemic conditions and is therefore protective against ischemic injury in lower limbs (35). Furthermore, Chen et al. demonstrated that endothelial NOX4 mediates angiogenesis (36). Thus, the presence of NOX4 in endothelial cells may induce a difference in phenotype between cardiac-specific NOX4 KO mice and systemic NOX4 KO mice. In addition, different models of PO may contribute to the difference in outcomes in the 2 studies. We conducted TAC in mice, whereas Zhang et al. induced aortic constriction by suprarenal banding. It is possible that the PO induced by suprarenal banding may be milder than that induced by TAC.

NOX4 activity was regulated by FYN not only in mitochondria but also in the ER and nucleus. FYN also regulated downstream subcellular compartment-specific targets of NOX4 such as HDAC4 and aconitase 2 in these fractions. Thus, the beneficial effects of overexpression of FYN are partially due to modification of these downstream signaling pathways of NOX4. Moreover, FYN regulated NOX4 activity during GD, thereby affecting autophagy and cell survival. These data indicate that FYN also negatively regulates the physiological function of NOX4; thus, negative regulation of NOX4 can be detrimental for cell survival under some conditions.

We here show that the levels of FYN expression and NOX4 phosphorylation at Y566 are lower in DCM hearts than in control hearts. We speculate that the downregulation of FYN may contribute to increases in oxidative stress in DCM hearts through derepression of NOX4. However, considering the fact that the function 
of NOX4 is context dependent, further investigation is required to elucidate the functional significance of the downregulation of FYN in human DCM.

In conclusion, we here demonstrate a potentially novel signaling mechanism controlling NOX4 activity and cardiac cell death. Cardiac stress activates/upregulates the nonreceptor-type tyrosine kinase FYN, which in turn negatively regulates NOX4 activity through phosphorylation of Y566 and prevents apoptosis in cardiac remodeling. FYN is a physiological regulator of NOX4 in the heart and, thus, the activity of NOX4 may be therapeutically controlled by targeted modulation of FYN.

\section{Methods}

Yeast two-hybrid screening. We used the yeast two-hybrid system (Clontech) and a human heart cDNA library. The cytosolic tail of mouse NOX4 (amino acids 406-578) served as bait.

Mice. cNOX4 KO mice have been described (6). CM-specific deletion of NOX4 was achieved using $\alpha$-myosin heavy chain-Cre (6). Mice with cardiac-specific overexpression of WT NOX4 (Tg-NOX4) were generated as described (2). Systemic FYN KO mice were purchased from Jackson Laboratory (stock number 012468). We generated mice with cardiac-specific overexpression of wild-type FYN (Tg-FYN) using the $\alpha$-myosin heavy chain promoter. We generated a genetic cross between cNOX4 KO and FYN KO mice. Bigenic mice were generated by crossing Tg-FYN mice with Tg-NOX4 mice. All experiments were conducted on 2- to 3-month-old male mice. All animal protocols were approved by the Institutional Animal Care and Use Committee of Rutgers New Jersey Medical School.

Primary culture of neonatal rat ventricular CMs. Primary cultures of ventricular CMs were prepared from 1-day-old Crl:(WI) BR-Wistar rats (Harlan). A CM-rich fraction was obtained by centrifugation through a discontinuous Percoll gradient as described (37).

Adenoviruses. Adenovirus vectors NOX4-HA, shRNA-NOX4 (Ad-shNOX4), shRNA-FYN (Ad-shFYN), shRNA-Src (Ad-shSrc), shRNA-YES (Ad-shYES), NOX4 Y566A-HA (Ad-NOX4 Y566A), NOX4 Y566D-HA (Ad-NOX4 Y566D), HyPer-mito (Ad-HyPer-mito) and FYN(1-80)-HA (Ad-FYN ${ }_{1-80} \mathrm{HA}$ ) were generated using the AdMax system (Microbix) as described (2). Adenovirus vectors harboring LacZ (Ad-LacZ) and sh-Scramble (Ad-shScr) were used as controls. Ad-FYN was purchased from Vector Biolab. The use of adenoviruses was approved by the Institutional Biosafety Committee of Rutgers New Jersey Medical School.

Recombinant proteins and pull-down binding assays. Proteins fused to hexameric histidine (His-tag) were expressed in E. coli strain BL21 (DE3) and purified with Ni beads. Proteins fused to S-protein (S-tag) were expressed using TnT Coupled Reticulocyte Lysate Systems purchased from Promega.

TAC. The methods used to impose PO in mice have been described (38). Mice were anesthetized with a mixture of ketamine $(0.065 \mathrm{mg} / \mathrm{g})$, xylazine $(0.013 \mathrm{mg} / \mathrm{g})$, and acepromazine $(0.002 \mathrm{mg} / \mathrm{g})$ and mechanically ventilated. The left side of the chest was opened at the second intercostal space. Aortic constriction was performed by ligation of the transverse thoracic aorta between the innominate artery and left common carotid artery with a 28-gauge needle using a 7-0 braided polyester suture. Sham operation was performed without constricting the aorta. To measure arterial pressure gradients, high-fidelity micromanometer catheters (1.4 French; Millar Instruments Inc.) were used.
Echocardiography. Mice were anesthetized using $12 \mu \mathrm{l} / \mathrm{g}$ body weight of $2.5 \%$ Avertin (Sigma-Aldrich), and echocardiography was performed using ultrasonography (Acuson Sequoia C256; Siemens Medical Solutions). A 13-MHz linear ultrasound transducer was used (38).

Immunostaining. Neonatal rat CMs grown on chamber slides (Lab-Tek) were washed 3 times with PBS. The cells were fixed with $4 \%$ paraformaldehyde and washed 4 times with PBS containing $0.1 \%$ Triton X-100. The cells were boiled for 10 minutes with a pressure cooker to allow better exposure of the antigen to the antibody. The cells were then blocked with PBS containing 5\% normal goat serum for 60 minutes and stained with antibodies as indicated. Images of NOX4 staining together with FYN and DAPI staining were obtained with confocal microscopy.

Immunoblot analyses. Heart homogenates and CM lysates were prepared in RIPA lysis buffer containing $50 \mathrm{mM}$ Tris (pH 7.5), $150 \mathrm{mM}$ $\mathrm{NaCl}, 1 \%$ IGEPAL CA-630 (Sigma Aldrich), 0.1\% SDS, 0.5\% deoxycholic acid, $10 \mathrm{mM} \mathrm{Na} \mathrm{P}_{2} \mathrm{O}_{7}, 5 \mathrm{mM}$ EDTA, $0.1 \mathrm{mM} \mathrm{Na}_{3} \mathrm{VO}_{4}, 1 \mathrm{mM} \mathrm{NaF}$, $0.5 \mathrm{mM}$ 4-(2-aminoethyl) benzenesulfonyl fluoride hydrochloride (AEBSF), $0.5 \mu \mathrm{g} / \mathrm{ml}$ aprotinin, and $0.5 \mu \mathrm{g} / \mathrm{ml}$ leupeptin. For immunoblot analyses, we used monoclonal antibodies raised against NOX4, as previously described (2), GAPDH (catalog G8795), tubulin (catalog T6074) (both Sigma-Aldrich), COX IV (catalog ab14744; Abcam), and polyclonal antibodies raised against FYN (catalog sc-28791), Src (catalog sc-5266), YES (catalog sc-46674), and p22 phox (catalog sc-20781) (all Santa Cruz Biotechnology), phospho-Src (Tyr416) (catalog 2101), His-tag (catalog 2366), HA-tag (catalog 3724), and S-tag (catalog 8476) (all Cell Signaling Technology). For detection of phosphorylation of NOX4 at Tyr566, a rabbit polyclonal phosphorylation-specific antibody was raised against a synthetic peptide of the C-terminus of mouse NOX4, SNRNNS(pTYR)GTKFEYC (GenScript).

Histological analyses. The LV accompanied by the septum was cut into base, middle portion, and apex, fixed with $10 \%$ formalin, embedded in paraffin, and sectioned at $6-\mu \mathrm{m}$ thickness. The sections were incubated in $3 \% \mathrm{H}_{2} \mathrm{O}_{2}$ in PBS to prevent endogenous peroxidation and blocked with 5\% BSA in PBS. CM cross-sectional area was measured from images captured of sections stained with anti-wheat germ agglutinin antibody as previously described (38). The outlines of $200 \mathrm{CMs}$ were traced in each section. Interstitial fibrosis was evaluated by Masson trichrome staining.

Apoptosis assays. TUNEL staining was conducted as described (39). Deparaffinized sections were incubated with proteinase K, and DNA fragments were labeled with fluorescein-conjugated dUTP using TdT (Roche Molecular Biochemicals). Nuclear density was determined by manual counting of DAPI-stained nuclei in 6 fields for each animal using a $40 \times$ objective.

Lucigenin chemiluminescence assay. Mitochondrial, ER, or nuclear fractions were suspended in $200 \mu \mathrm{l}$ of an assay buffer composed of $100 \mathrm{mM}$ potassium phosphate ( $\mathrm{pH}$ 7.0), $10 \mu \mathrm{M}$ flavin adenine dinucleotide, $1 \mathrm{mM} \mathrm{NaN}_{3}$, and $1 \mathrm{mM} \mathrm{EGTA}$. After preincubation with $5 \mu \mathrm{M}$ lucigenin, NADH or NADPH was added to a final concentration of $500 \mu \mathrm{M}(40)$. The chemiluminescence was continuously monitored using a luminometer. The reaction was terminated by the addition of SOD $(100 \mu \mathrm{g} / \mathrm{ml})(6)$. Rotenone (10 $\mathrm{nM}$, Sigma-Aldrich) was added to mitochondrial fractions 5 minutes before the addition of NADPH.

Viability of the cells. Cell viability was measured by Cell Titer Blue (CTB) assays (Promega). In brief, CMs $\left(1 \times 10^{5}\right.$ per $\left.100 \mu \mathrm{l}\right)$ were seeded onto 96-well dishes. The cells were preincubated with the indicated 
adenovirus for 48 hours and then the cells were cultured with normal or glucose-free medium for 24 hours. Viable cell numbers were measured by CTB assays according to the supplier's protocol.

Amplex Red assay. $\mathrm{H}_{2} \mathrm{O}_{2}$ production was measured with a Molecular Probes Amplex Red $\mathrm{H}_{2} \mathrm{O}_{2}$ assay kit (Invitrogen) as described (4). 1-Naphthyl PP1, a FYN inhibitor, was purchased from Santa Cruz Biotechnology (sc-203765). CMs were treated with 1-naphthyl PP1 (0, 0.3, 1,3 , and $10 \mu \mathrm{M}$ ) for 30 minutes. To measure $\mathrm{H}_{2} \mathrm{O}_{2}$ derived from Nox, mitochondrial, ER, or nuclear fractions were incubated with $50 \mu \mathrm{M}$ Amplex Red solution for 10 minutes after the addition of NADPH.

Human samples from explanted hearts. The samples from explanted hearts used in this study were obtained from 4 patients who had received heart transplants and 4 age-matched donors at the Taipei Veterans General Hospital. The study was approved by the Institutional Ethics Committee at the Taipei Veterans General Hospital (VGHIRB no. 2012-06-028D), and all patients or their families expressed their willingness to participate through an informed consent form. Myocardial posterior wall samples were collected during preparation of donor hearts for transplantation in the hospital. Myocardial samples from near the mitral annulus were obtained from recipients at the time of therapeutic transplantation. Immediately after tissue procurement, the samples for biochemical study were stored in liquid nitrogen and kept at $-80^{\circ} \mathrm{C}$.

In vitro kinase assays. Active FYN (10 ng) was incubated with recombinant NOX4-CT $(1 \mu \mathrm{g})$ in a kinase buffer (50 mM HEPES [pH 7.4], $15 \mathrm{mM} \mathrm{MgCl}_{2}$, and $200 \mu \mathrm{M}$ sodium vanadate containing 100 $\mu \mathrm{M}$ ATP and $10 \mu \mathrm{Ci}\left[\gamma^{-32} \mathrm{P}\right]$ ATP per reaction) at $30^{\circ} \mathrm{C}$ for 30 minutes. Phosphorylated proteins were resolved by SDS-PAGE and analyzed by autoradiography. Active FYN (10 ng) was also incubated with NOX4CT peptides $(1 \mu \mathrm{g})$ in the same kinase buffer. Six peptides originating from NOX4-CT were examined: NOX4-CT-338 (PQQYILHCP), NOX4-CT-404-408 (RNYPKLYID), NOX4-CT-423 (SLNYEVSLC), NOX4-CT-452 (DWKPYKLRR), NOX4-CT-490 (FVNIQLYLSQ), and NOX4-CT-566 (NRNNSYGTKF).

Mass spectrometry. Coomassie blue-stained gel bands were excised and washed 3 times with $30 \%$ acetonitrile (ACN) in $50 \mathrm{mM}$ ammonium bicarbonate for destaining. The protein was first reduced by $10 \mathrm{mM}$ DTT and then alkylated by $50 \mathrm{mM}$ iodoacetamide prior to trypsin digestion at $37^{\circ} \mathrm{C}$ overnight. The resulting peptides were analyzed by liquid chromatography-tandem mass spectrometry (LC-MS/MS) on an Ultimate 3000 (Dionex) LC system coupled with an Orbitrap Velos instrument (Thermo Fisher Scientific). In brief, the peptides were resolved by a C18 reversed-phase column $(75 \mu \mathrm{m} \times 150$ mm, $3 \mu \mathrm{m}, 100 \AA$ A, C18; Dionex) using a 95-minute gradient with solvent $\mathrm{A}(2 \% \mathrm{ACN}, 0.1 \%$ formic acid $[\mathrm{FA}])$ and solvent B (85\% $\mathrm{ACN}$,
$0.1 \% \mathrm{FA}): 60$ minutes from $3 \%$ to $25 \% \mathrm{~B}, 15$ minutes from $25 \%$ to $45 \% \mathrm{~B}$, and 10 minutes from $45 \%$ to $95 \% \mathrm{~B}$. The eluted peptides were introduced into a nano-ESI source with a spray voltage of $2 \mathrm{kV}$ and a capillary temperature of $275^{\circ} \mathrm{C}$. MS spectra were acquired in positive mode with an $m / z$ scan range of 350-2,000. The 10 most abundant ions were selected for CID fragmentation with an isolation width of 2 $\mathrm{m} / \mathrm{z}$ and a minimal ion fragmentation threshold of 3,000. A lock mass function was used for accurate mass measurement. The raw spectra were searched against the SWISS-PROT human database using the Mascot search engine on the Proteome Discoverer software platform (Thermo Fisher Scientific). The error window was set at $10 \mathrm{ppm}$ for MS and 0.5 Da for MS/MS. Cysteine carbamidomethylation, methionine oxidation, and serine, threonine, and tyrosine phosphorylation were set as variable modifications. The resulting phosphopeptides and phosphorylation sites were manually evaluated.

Statistics. Data are expressed as the mean \pm SEM. Testing differences in means between 2 groups was done by unpaired $t$ tests, whereas those among more than 2 groups were by 1-way ANOVA followed by a post-hoc Fisher's comparison test. $P<0.05$ was considered to be statistically significant.

\section{Author contributions}

$\mathrm{SM}, \mathrm{JK}$, and JS conceived the study. SM, JK, SI, NN, SO, and CPH performed experiments. PZ conducted mouse surgeries and hemodynamic analyses. TL and HL conducted proteomic analyses. SK and HT provided regents and intellectual input. SM, JK, and TL performed data analyses. SM wrote the manuscript. JS commented extensively on the manuscript. All coauthors read and approved the final manuscript.

\section{Acknowledgments}

The authors thank Daniela Zablocki and Christopher D. Brady for critical reading of the manuscript. This work was supported in part by US Public Health Service Grants HL67724, HL91469, HL102738, HL112330, and AG23039 (to JS) and by the Leducq Foundation Transatlantic Network of Excellence (to JS). SM has been supported by a Postdoctoral Fellowship from the American Heart Association, Banyu Foundation Research Grant, Takeda Science Foundation, and Sakakibara Memorial Research Grant.

Address correspondence to: Junichi Sadoshima, Department of Cell Biology and Molecular Medicine, Cardiovascular Research Institute, Rutgers New Jersey Medical School, 185 South Orange Avenue, MSB G609, Newark, New Jersey 07103, USA. Phone: 973.972.8916; E-mail: sadoshju@njms.rutgers.edu.
1. Maejima Y, Kuroda J, Matsushima S, Ago T, Sadoshima J. Regulation of myocardial growth and death by NADPH oxidase. J Mol Cell Cardiol. 2011;50(3):408-416

2. Ago T, Kuroda J, Pain J, Fu C, Li H, Sadoshima J. Upregulation of Nox4 by hypertrophic stimuli promotes apoptosis and mitochondrial dysfunction in cardiac myocytes. Circ Res. 2010;106(7):1253-1264.

3. Matsushima S, et al. Increased oxidative stress in the nucleus caused by Nox 4 mediates oxidation of HDAC4 and cardiac hypertrophy. Circ Res.
2013;112(4):651-663.

4. Sciarretta S, et al. Activation of NADPH oxidase 4 in the endoplasmic reticulum promotes cardiomyocyte autophagy and survival during energy stress through the protein kinase RNA-activatedlike endoplasmic reticulum kinase/eukaryotic initiation factor $2 \alpha$ /activating transcription factor 4 pathway. Circ Res. 2013;113(11):1253-1264.

5. Zhao QD, et al. NADPH oxidase 4 induces cardiac fibrosis and hypertrophy through activating Akt/mTOR and NFкB signaling pathways. Circulation. 2015;131(7):643-655.
6. Kuroda J, Ago T, Matsushima S, Zhai P, Schnei der MD, Sadoshima J. NADPH oxidase 4 (Nox4) is a major source of oxidative stress in the failing heart. Proc Natl Acad Sci U S A. 2010;107(35):15565-15570.

7. Zhang M, et al. NADPH oxidase- 4 mediates protection against chronic load-induced stress in mouse hearts by enhancing angiogenesis. Proc Natl Acad Sci U S A. 2010;107(42):18121-18126.

8. Giordano FJ. Oxygen, oxidative stress, hypoxia, and heart failure. J Clin Invest. 2005;115(3):500-508. 
9. Finkel T. Oxidant signals and oxidative stress. Curr Opin Cell Biol. 2003;15(2):247-254.

10. Matsushima S, et al. Broad suppression of NADPH oxidase activity exacerbates ischemia/ reperfusion injury through inadvertent downregulation of hypoxia-inducible factor- $1 \alpha$ and upregulation of peroxisome proliferator-activated receptor- $\alpha$. Circ Res. 2013;112(8):1135-1149.

11. Abo A, Pick E, Hall A, Totty N, Teahan CG, Segal AW. Activation of the NADPH oxidase involves the small GTP-binding protein p21rac1. Nature. 1991;353(6345):668-670.

12. Uhlinger DJ, Taylor KL, Lambeth JD. p67-phox enhances the binding of $\mathrm{p} 47$-phox to the human neutrophil respiratory burst oxidase complex. J Biol Chem. 1994;269(35):22095-22098.

13. Geiszt M, Kopp JB, Várnai P, Leto TL. Identification of renox, an NAD(P)H oxidase in kidney. Proc Natl Acad Sci U S A. 2000;97(14):8010-8014.

14. Martyn KD, Frederick LM, von Loehneysen K, Dinauer MC, Knaus UG. Functional analysis of Nox4 reveals unique characteristics compared to other NADPH oxidases. Cell Signal. 2006;18(1):69-82.

15. von Löhneysen K, Noack D, Jesaitis AJ, Dinauer MC, Knaus UG. Mutational analysis reveals distinct features of the Nox4-p22 phox complex. J Biol Chem. 2008;283(50):35273-35282.

16. Giannoni E, Chiarugi P. Redox circuitries driving Src regulation. Antioxid Redox Signal. 2014;20(13):2011-2025.

17. Smyrnias I, et al. Nicotinamide adenine dinucleotide phosphate oxidase-4-dependent upregulation of nuclear factor erythroid-derived 2-like 2 protects the heart during chronic pressure overload. Hypertension. 2015;65(3):547-553.

18. McCord JM. Oxygen-derived free radicals in postischemic tissue injury. $N$ Engl JMed. 1985;312(3):159-163.

19. Ide T, et al. Direct evidence for increased hydrox$\mathrm{yl}$ radicals originating from superoxide in the failing myocardium. Circ Res. 2000;86(2):152-157.

20. Grieve DJ, et al. Involvement of the nicotinamide adenosine dinucleotide phosphate oxidase isoform Nox2 in cardiac contractile dysfunction occurring in response to pressure overload. J Am Coll Cardiol. 2006;47(4):817-826.

21. Matsuno K, et al. NOX1/NADPH oxidase is involved in endotoxin-induced cardiomyocyte apoptosis. Free Radic Biol Med. 2012;53(9):1718-1728.

22. Lyle AN, et al. Poldip2, a novel regulator of Nox4 and cytoskeletal integrity in vascular smooth muscle cells. Circ Res. 2009;105(3):249-259.

23. Palomero T, et al. Recurrent mutations in epigenetic regulators, RHOA and FYN kinase in peripheral T cell lymphomas. Nat Genet. 2014;46(2):166-170.

24. Um JW, et al. Alzheimer amyloid- $\beta$ oligomer bound to postsynaptic prion protein activates Fyn to impair neurons. Nat Neurosci. 2012;15(9):1227-1235.

25. Yamada E, Pessin JE, Kurland IJ, Schwartz GJ, Bastie CC. Fyn-dependent regulation of energy expenditure and body weight is mediated by tyrosine phosphorylation of LKB1. Cell Metab. 2010;11(2):113-124

26. Saito YD, Jensen AR, Salgia R, Posadas EM. Fyn: a novel molecular target in cancer. Cancer. 2010;116(7):1629-1637.

27. Tang X, Feng Y, Ye K. Src-family tyrosine kinase fyn phosphorylates phosphatidylinositol 3-kinase enhancer-activating Akt, preventing its apoptotic cleavage and promoting cell survival. Cell Death Differ. 2007;14(2):368-377.

28. Rosado JA, Redondo PC, Salido GM, GómezArteta E, Sage SO, Pariente JA. Hydrogen peroxide generation induces pp 60 src activation in human platelets: evidence for the involvement of this pathway in store-mediated calcium entry. J Biol Chem. 2004;279(3):1665-1675.

29. Reinehr R, Becker S, Eberle A, Grether-Beck S, Häussinger D. Involvement of NADPH oxidase isoforms and Src family kinases in CD95dependent hepatocyte apoptosis. J Biol Chem. 2005;280(29):27179-27194.

30. Cooper JA, Howell B. The when and how of Src regulation. Cell.1993;73(6):1051-1054.
31. Xu W, Harrison SC, Eck MJ. Three-dimensional structure of the tyrosine kinase c-Src. Nature. 1997;385(6617):595-602.

32. Giannoni E, Buricchi F, Raugei G, Ramponi G, Chiarugi P. Intracellular reactive oxygen species activate Src tyrosine kinase during cell adhesion and anchorage-dependent cell growth. Mol Cell Biol. 2005;25(15):6391-6403.

33. Yadav V, Denning MF. Fyn is induced by Ras/PI3K/Akt signaling and is required for enhanced invasion/migration. Mol Carcinog. 2011;50(5):346-352.

34. Ninio-Many L, Grossman H, Shomron N, Chuderland D, Shalgi R. microRNA-125a-3p reduces cell proliferation and migration by targeting Fyn. JCell Sci. 2013;126(Pt 13):2867-2876.

35. Di Bartolo BA, et al. Tumor necrosis factor-related apoptosis-inducing ligand (TRAIL) promotes angiogenesis and ischemia-induced neovascularization via NADPH oxidase 4 (NOX4) and nitric oxide-dependent mechanisms. JAm Heart Assoc. 2015;4(11): e002527.

36. Chen L, et al. Both hydrogen peroxide and transforming growth factor beta 1 contribute to endothelial Nox4 mediated angiogenesis in endothelial Nox4 transgenic mouse lines. Biochim Biophys Acta. 2014;1842(12 Pt A):2489-2499.

37. Morisco C, Zebrowski D, Condorelli G, Tsichlis P, Vatner SF, Sadoshima J. The Akt-glycogen synthase kinase 3beta pathway regulates transcription of atrial natriuretic factor induced by beta-adrenergic receptor stimulation in cardiac myocytes. J Biol Chem. 2000;275(19):14466-14475.

38. Matsuda T, et al. Distinct roles of GSK-3alpha and GSK-3beta phosphorylation in the heart under pressure overload. Proc Natl Acad Sci USA. 2008;105(52):20900-20905.

39. Yamamoto S, et al. Activation of Mst1 causes dilated cardiomyopathy by stimulating apoptosis without compensatory ventricular myocyte hypertrophy. JClin Invest. 2003;111(10):1463-1474.

40. Ago T, et al. Nox4 as the major catalytic component of an endothelial NAD(P)H oxidase. Circulation. 2004;109(2):227-233. 